\title{
Boundary optimal feedback controller for time-periodic Stokes-Oseen flows
}

\author{
Hanbing Liu
}

\begin{abstract}
This work considered the Dirichlet boundary optimal control of time-periodic Stokes-Oseen equations. The existence of optimal solution and maximum principle are obtained without assuming that the normal component of the control is equal to zero. Moreover, we get the regularity result of the optimal solution via the Euler-Lagrange system. The existence of solution to the HJB equation is proved. The feedback form of the optimal controller is given, and with this feedback controller, we can get for the solution to the periodic Navier-Stokes equations the property of continuous dependence on the outer force term.
\end{abstract}

Mathematics Subject Classification (2010). 49A22, 49L20, 49N35, 76D07.

Keywords. Dirichlet boundary control, Time-periodic Stokes-Oseen equations, Feedback controller.

\section{Introduction}

Here we shall consider the optimal control problem:

$$
\left(P_{0}\right) \quad \min \left\{\frac{1}{2} \int_{0}^{T} \int_{\Omega}|\mathbf{y}|^{2} d \mathbf{x} d t+\frac{1}{2} \int_{0}^{T} \int_{\Gamma}|\mathbf{u}|^{2} d \mathbf{x} d t\right\}
$$

subject to the periodic Stokes-Oseen equations with Dirichlet boundary condition:

$$
\begin{cases}\frac{d \mathbf{y}}{d t}-\nu \triangle \mathbf{y}+\left(\mathbf{f}_{1} \cdot \nabla\right) \mathbf{y}+(\mathbf{y} \cdot \nabla) \mathbf{f}_{2}+\nabla p=\mathbf{f}_{0} & \text { in } \Omega \times(0, T) \\ \mathbf{y}(0)=\mathbf{y}(T) & \text { in } \Omega, \\ \nabla \cdot \mathbf{y}=0 & \text { in } \Omega \times(0, T) \\ \mathbf{y}=\mathbf{u} & \text { on } \Gamma \times(0, T)\end{cases}
$$

This work was supported by "the Fundamental Research Funds for the Central Universities", No.CUG130620. It was also partially supported by National Basic Research Program of China (973 Program), No.2010CB950904, No.2011CB710604, by the Marie Curie ITN on "Controlled Systems", No. 213841/2008. 
Here $\Omega$ is a bounded open subset with smooth boundary $\Gamma$ in $\mathbb{R}^{2}$. Function $\mathbf{u}(t) \in U, \forall t \in(0, T)$ is the boundary control, where $U=V^{0}(\Gamma) \doteq\{\mathbf{u} \in$ $\left.\left(L^{2}(\Gamma)\right)^{2} ;\langle\mathbf{u} \cdot \mathbf{n}, 1\rangle_{\left(\left(H^{-1 / 2}(\Gamma)\right)^{2},\left(H^{1 / 2}(\Gamma)\right)^{2}\right)}=0\right\}$. Here $\mathbf{n}$ is the outer normal vector of $\Gamma, \mathbf{f}_{i}(\mathbf{x}), i=1,2$ are steady state functions and $\mathbf{f}_{i} \in\left(W^{2, \infty}(\Omega)\right)^{2} \cap V^{0}(\Omega)$, where space $V^{0}(\Omega)$ is defined by

$$
V^{0}(\Omega)=\left\{\mathbf{y} \in\left(L^{2}(\Omega)\right)^{2} ; \nabla \cdot \mathbf{y}=0 \text { in } \Omega,\langle\mathbf{y} \cdot \mathbf{n}, 1\rangle_{\left(\left(H^{-1 / 2}(\Gamma)\right)^{2},\left(H^{1 / 2}(\Gamma)\right)^{2}\right)}=0\right\} .
$$

In the above definition and throughout what follows, for all $\mathbf{y} \in\left(L^{2}(\Omega)\right)^{2}$ such that $\nabla \cdot \mathbf{y} \in\left(L^{2}(\Omega)\right)^{2}$, we denote by $\mathbf{y} \cdot \mathbf{n}$ the normal trace of $\mathbf{y}$ in the space $\left(H^{-1 / 2}(\partial \Omega)\right)^{2}$ (see $\left.[3]\right)$.

In fluid dynamics, the Stokes-Oseen equations (or Stokes-Oseen flow) describe the flow of a viscous and incompressible fluid at small Reynolds numbers. This is a typical situation in flows where the fluid velocities are very slow, the viscosities are very large, or the length-scales of the flow are very small. In nature this type of flow occurs in the swimming of microorganisms, the flow of lava and so on. In technology, it occurs in paint, microelectromechanical systems devices, and in the flow of viscous polymers generally.

For the optimal control problems of periodic flows we cite here $[2,13,14]$ and the references in [21]. In [14], the optimal feedback controllers are constructed for the periodic convex control problems. Dirichlet boundary optimal control problems of Stokes-Oseen equations with initial data are usually considered in the research of the boundary stabilization of Navier-Stokes equations. Here we cite works of this domain Barbu [1,15], Raymond [18,20], and the references therein.

In this work, since the normal component of the boundary control is not 0 , we shall apply the method in [18] to decompose the state equation, satisfied by the velocity field $\mathbf{y}$ to be an evolution equation satisfied by $P \mathbf{y}$, where $P$ is the so-called Leray projection operator, and a quasi-stationary elliptic equation satisfied by $(I-P) \mathbf{y}$. Using this decomposition we can derive the optimality system of optimal control problem $\left(P_{0}\right)$. With the Euler-Lagrange system, and a sequence of regularity results obtained for periodic solution to Stokes equations, which are listed in the appendix, we can improve the regularity of the optimal solution. For the purpose to focus on the idea of approaching the feedback controller, we shall consider control problem $\left(P_{0}\right)$ in the second part of this work (Sect. 3) with the tangential boundary control without lose of generality (the feedback law can be expressed only in function of $P \mathbf{y}$, see [18]). Thanks to the regularity results obtained for the optimal solution, we can get the existence of solution to the HJB equation by following the method applied in [14], wherein the feedback optimal form is obtained for internal periodic control. However, since the optimal control problem considered here is unbounded, the approach will be more precise and difficult. Consequently, we can get the boundary feedback optimal controller. Finally, we shall apply this feedback operator to the periodic Navier-Stokes equations. This idea is originated as following: when the stationary Navier-Stokes equations are perturbed by a small time-periodic outer force, the perturbed periodic solution to the Navier-Stokes equations may do not stay near the original solution, 
even when the perturbation is very small; to overcome this defect, we can put the boundary feedback controller in the perturbed periodic Navier-Stokes equations, and we can show that for the nonlinear system with this feedback controller, the periodic solution is continuously dependent on the outer force term.

The space $V^{0}(\Omega)$ is a closed subspace of $\left(L^{2}(\Omega)\right)^{2}$, and it is a Hilbert space with the scalar product

$$
\langle\mathbf{y}, \mathbf{z}\rangle=\int_{\Omega} \mathbf{y} \cdot \mathbf{z} d \mathbf{x}
$$

and the corresponding norm $|\mathbf{y}|=\left(\int_{\Omega}|\mathbf{y}|^{2} d \mathbf{x}\right)^{1 / 2}$. We shall denote by the same symbol $|\cdot|$ the norms in $\mathbb{R}^{2}, L^{2}(\Omega)^{2}$ and $V^{0}(\Omega)$. The scalar product in $\left(L^{2}(\Omega)\right)^{2}$ is the same as in $V^{0}(\Omega)$, which will be also denoted by $\langle\cdot, \cdot\rangle$ if there is no ambiguous. Define the spaces

$$
\begin{gathered}
V^{s}(\Omega)=\left(H^{s}(\Omega)\right)^{2} \cap V^{0}(\Omega), \quad s \geq 0, \\
V_{n}^{s}(\Omega)=\left\{\mathbf{y} \in\left(H^{s}(\Omega)\right)^{2}, \nabla \cdot \mathbf{y}=0 \text { in } \Omega, \mathbf{y} \cdot \mathbf{n}=0 \text { on } \Gamma\right\}, \quad s \geq 0, \\
V_{0}^{s}(\Omega)=\left\{\mathbf{y} \in\left(H^{s}(\Omega)\right)^{2}, \nabla \cdot \mathbf{y}=0 \text { in } \Omega, \mathbf{y}=0 \text { on } \Gamma\right\}, \quad s \geq 1 / 2, \\
V^{s}(\Gamma)=\left\{\mathbf{u} \in\left(H^{s}(\Gamma)\right)^{2},\langle\mathbf{u} \cdot \mathbf{n}, 1\rangle_{\left(\left(H^{-1 / 2}(\Gamma)\right)^{2},\left(H^{1 / 2}(\Gamma)\right)^{2}\right)}=0\right\}, \quad s \geq-1 / 2, \\
V_{n}^{s}(\Gamma)=\left\{\mathbf{u} \in V^{s}(\Gamma), \mathbf{u} \cdot \mathbf{n}=0\right\}, \quad s \geq-1 / 2,
\end{gathered}
$$

where $H^{s}(\Omega), H^{s}(\Gamma)$ are the Sobolev spaces. The norms of spaces $\left(H^{s}(\Omega)\right)^{2}$ and $V^{s}$ will be denoted by $\|\cdot\|_{s}$. The norm $\|\cdot\|_{1}$ will denote by $\|\cdot\|$ for simplicity. We shall also be denoted by $|\cdot|$ and $\langle\cdot, \cdot\rangle$ the norm and inner product respectively of spaces $L^{2}(\Gamma), V^{0}(\Gamma)$ and $V_{n}^{0}(\Gamma)$. We shall use the notations $Q_{T}=\Omega \times(0, T), \Sigma_{T}=\Gamma \times(0, T)$. For spaces of time dependent functions we set

$$
\begin{aligned}
& V^{s, \sigma}\left(Q_{T}\right)=H^{\sigma}\left(0, T ; V^{0}(\Omega)\right) \cap L^{2}\left(0, T ; V^{s}(\Omega)\right), \\
& V^{s, \sigma}\left(\Sigma_{T}\right)=H^{\sigma}\left(0, T ; V^{0}(\Sigma)\right) \cap L^{2}\left(0, T ; V^{s}(\Sigma)\right) .
\end{aligned}
$$

Define the trilinear function

$$
b(\mathbf{y}, \mathbf{z}, \mathbf{w})=\sum_{i, j=1}^{2} \int_{\Omega} y_{i} \frac{\partial}{\partial x_{i}} z_{j} w_{j}, \quad \forall \mathbf{y}, \mathbf{z}, \mathbf{w} \in V^{1}(\Omega) .
$$

We have the following properties for the trilinear function $b$ (See proposition 5.14 in [4]):

$$
\begin{aligned}
& b(\mathbf{y}, \mathbf{z}, \mathbf{w})=-b(\mathbf{y}, \mathbf{w}, \mathbf{z}), \quad \forall y, z, w \in V \\
& |b(\mathbf{y}, \mathbf{z}, \mathbf{w})| \leq C\|\mathbf{y}\|_{m_{1}}\|\mathbf{z}\|_{m_{2}}\|\mathbf{w}\|_{m_{3}}, \quad \forall \mathbf{y} \in V_{m_{1}}, \mathbf{z} \in V_{m_{2}}, \mathbf{w} \in V_{m_{3}},
\end{aligned}
$$

where $m_{i} \geq 0, i=1,2,3$, and

$$
\begin{array}{ll}
m_{1}+m_{2}+m_{3} \geq 1, \text { if } m_{i} \neq 1, & \forall i=1,2,3, \\
m_{1}+m_{2}+m_{3}>1, \text { if } m_{i}=1, & \text { for some } i=1,2,3 .
\end{array}
$$

Here $V_{m_{i}}=V \cap\left(H_{0}^{m_{i}}(\Omega)\right)^{2}$. 
We shall denote by $P:\left(L^{2}(\Omega)\right)^{2} \rightarrow V_{n}^{0}(\Omega)$ the orthogonal projection of $\left(L^{2}(\Omega)\right)^{2}$ onto $V_{n}^{0}(\Omega)$, and set $A=-P \Delta, D(A)=V \cap\left(H^{2}(\Omega)\right)^{2}$. Equivalently,

$$
\langle A \mathbf{y}, \mathbf{z}\rangle=a(\mathbf{y}, \mathbf{z})=\int_{\Omega} \nabla \mathbf{y} \cdot \nabla \mathbf{z} d \mathbf{x}, \quad \forall \mathbf{y}, \mathbf{z} \in V .
$$

The stokes operator $A$ is self-adjoint in $H, A \in L\left(V, V^{\prime}\right)\left(V^{\prime}\right.$ is the dual of $V$ with the norm denoted by $\left.\|\cdot\|_{V^{\prime}}\right)$ and $\langle A \mathbf{y}, \mathbf{y}\rangle=\|\mathbf{y}\|^{2}, \forall \mathbf{y} \in V$. Denote by $A_{0}$ the operator defined by $A_{0} \mathbf{y}=P\left(\left(\mathbf{f}_{1} \cdot \nabla\right) \mathbf{y}+(\mathbf{y} \cdot \nabla) \mathbf{f}_{2}\right)$. Then operator $\mathcal{A}=$ $\nu A+A_{0}: D(\mathcal{A})\left(=V \cap\left(H^{2}(\Omega)\right)^{2}\right) \rightarrow V_{n}^{0}(\Omega)$ can be defined on the space $V_{n}^{0}(\Omega)$ with image in space $\left(D\left(\mathcal{A}^{*}\right)\right)^{\prime}$, which is the dual space of $D\left(\mathcal{A}^{*}\right)(=D(\mathcal{A}))$, by transposition

$$
\langle\mathcal{A} \mathbf{y}, \mathbf{z}\rangle_{\left(\left(D\left(\mathcal{A}^{*}\right)\right)^{\prime}, D\left(\mathcal{A}^{*}\right)\right)}=\left\langle\mathbf{y}, \mathcal{A}^{*} \mathbf{z}\right\rangle, \quad \forall \mathbf{z} \in D\left(\mathcal{A}^{*}\right) .
$$

Here $\langle\cdot, \cdot\rangle_{\left(\left(D\left(\mathcal{A}^{*}\right)\right)^{\prime}, D\left(\mathcal{A}^{*}\right)\right)}$ denotes the dual pair of the space $\left(D\left(\mathcal{A}^{*}\right)\right)^{\prime}$ and $D\left(\mathcal{A}^{*}\right)$. We still denote by $\mathcal{A}$ the extended operator. The operator $\mathcal{A}^{*}$ is the adjoint operator of $\mathcal{A}$ which is defined by

$$
\mathcal{A}^{*} \mathbf{z}=A \mathbf{z}+P\left(-\left(\mathbf{f}_{1} \cdot \nabla\right) \mathbf{z}+\mathbf{z} \cdot\left(\nabla \mathbf{f}_{2}\right)^{T}\right), \quad \forall \mathbf{z} \in D(\mathcal{A}) .
$$

Define the operator $D:\left(H^{s}(\Gamma)\right)^{2} \rightarrow\left(H^{\frac{1}{2}+s}(\Omega)\right)^{2}, s \geq 1 / 2$, by the solution $\mathbf{z}=D \mathbf{u}$ to the following equation

$$
\begin{cases}k \mathbf{z}-\nu \triangle \mathbf{z}+\left(\mathbf{f}_{\mathbf{1}} \cdot \nabla\right) \mathbf{z}+(\mathbf{z} \cdot \nabla) \mathbf{f}_{\mathbf{2}}+\nabla p_{1}=0 & \text { in } \Omega, \\ \operatorname{div} \mathbf{z}=0 & \text { in } \Omega, \\ \mathbf{z}=\mathbf{u} & \text { on } \\ \Gamma, & \end{cases}
$$

where $k>0$ is an element in the resolvent set of $-\mathcal{A}$ such that $\langle(k I+\mathcal{A}) \mathbf{y}, \mathbf{y}\rangle \geq$ $\omega_{0}\|\mathbf{y}\|^{2}, \forall \mathbf{y} \in D(A)$, for some $0<\omega_{0}<1$.

For all $\psi \in H^{1 / 2+\varepsilon}(\Omega)$ with $\varepsilon>0$, we denote by $c(\psi)$ the constants defined by

$$
c(\psi)=\frac{1}{|\Gamma|} \int_{\Gamma} \psi .
$$

The results presented below can be referred to the work of Raymond [18].

Lemma 1.1. (i) The operator $D$ is a bounded operator from $V^{0}(\Gamma)$ into $V^{0}(\Omega)$, moreover, it satisfies

$$
\|D \mathbf{u}\|_{V^{s+1 / 2}(\Omega)} \leq C(s)\|\mathbf{u}\|_{V^{s}(\Omega)}, \text { for all } 0 \leq s \leq 2 .
$$

(ii) The operator $D^{*} \in \mathcal{L}\left(V^{0}(\Omega), V^{0}(\Gamma)\right)$, is defined by

$$
D^{*} \mathbf{g}=-\nu \frac{\partial \mathbf{z}}{\partial \mathbf{n}}+\pi \mathbf{n}-c(\pi) \mathbf{n}
$$

where $(\mathbf{z}, \pi)$ is the solution of

$$
\lambda_{0} \mathbf{z}-\nu \Delta \mathbf{z}+\left(\mathbf{f}_{\mathbf{1}} \cdot \nabla\right) \mathbf{z}+\left(\nabla \mathbf{f}_{\mathbf{2}}\right)^{T} \mathbf{z}+\nabla \pi=\mathbf{g}, \operatorname{div} \mathbf{z}=0,\left.\mathbf{z}\right|_{\Gamma}=0,
$$

and $c(\pi)$ is defined by (1.3).

Let us define the operators $\gamma_{\tau} \in \mathcal{L}\left(V^{0}(\Gamma)\right), \gamma_{n} \in \mathcal{L}\left(V^{0}(\Gamma)\right)$ by

$$
\gamma_{\tau} \mathbf{u}=\mathbf{u}-(\mathbf{u} \cdot \mathbf{n}) \mathbf{n}, \gamma_{n} u=(\mathbf{u} \cdot \mathbf{n}) \mathbf{n}=\mathbf{u}-\gamma_{\tau} \mathbf{u} .
$$


Lemma 1.2. The operators $\gamma_{\tau}$ and $\gamma_{n}$ satisfy

$$
\gamma_{\tau}=\gamma_{\tau}^{*}, \gamma_{n}=\gamma_{n}^{*} \text {, and }(I-P) D=(I-P) D \gamma_{n} .
$$

Lemma 1.3. The operator

$$
R_{A}=D^{*}(I-P) D+I
$$

is an isomorphism from $V^{0}(\Gamma)$ into itself. Moreover, for all $0 \leq s \leq 3 / 2$, its restriction to $V^{s}(\Gamma)$ is an isomorphism from $V^{s}(\Gamma)$ into itself. In addition $R_{A}$ satisfies

$$
R_{A} \gamma_{n}=\gamma_{n} R_{A} \gamma_{n}, R_{A} \gamma_{\tau}=\gamma_{\tau} R_{A} \gamma_{\tau}=\gamma_{\tau}
$$

The restriction of $R_{A}$ to $V_{\tau}^{0}(\Gamma)$ is an isomorphism from $V_{\tau}^{0}(\Gamma)$ into itself, and we have

$$
R_{A}^{-1} \mathbf{u}=\left(\gamma_{n} R_{A} \gamma_{n}\right)^{-1} \mathbf{u}=\gamma_{n} R_{A}^{-1} \mathbf{u}, \quad \forall \mathbf{u} \in V_{\tau}^{0}(\Gamma)
$$

We introduce the operators $F_{n}=\mathcal{A}_{k} P D \gamma_{n}, F_{\tau}=\mathcal{A}_{k} P D \gamma_{\tau}, F=F_{n}+F_{\tau}$. where $\mathcal{A}_{k}=k I+\mathcal{A}$.

Proposition 1.4. For all $\boldsymbol{\Phi} \in D(A), F^{*} \boldsymbol{\Phi} \in V^{1 / 2}(\Gamma)$, we have

$$
F^{*} \boldsymbol{\Phi}=D^{*} \mathcal{A}_{k}^{*} \Phi, F_{\tau}^{*} \boldsymbol{\Phi}=\gamma_{\tau} D^{*} \mathcal{A}_{k}^{*} \boldsymbol{\Phi}, F_{n}^{*} \boldsymbol{\Phi}=\gamma_{n} D^{*} \mathcal{A}_{k}^{*} \mathbf{\Phi},
$$

and

$$
F^{*} \boldsymbol{\Phi}=-\nu \frac{\partial \boldsymbol{\Phi}}{\partial \mathbf{n}}+\psi \mathbf{n}-c(\psi) \mathbf{n}, F_{\tau}^{*} \boldsymbol{\Phi}=-\nu \frac{\partial \boldsymbol{\Phi}}{\partial \mathbf{n}}, F_{n}^{*} \Phi=\psi \mathbf{n}-c(\psi) \mathbf{n},
$$

with

$$
\nabla \psi=(I-P)\left(\nu \triangle \boldsymbol{\Phi}+\left(\mathbf{f}_{\mathbf{1}} \cdot \nabla\right) \boldsymbol{\Phi}-\left(\nabla \mathbf{f}_{\mathbf{2}}\right)^{T} \mathbf{\Phi}\right) .
$$

We rewrite Eq. (1.1) in the form

$$
\begin{gathered}
P \mathbf{y}^{\prime}+\mathcal{A} P \mathbf{y}=F \mathbf{u}+\mathbf{f}, \mathbf{y}(0)=\mathbf{y}(T), \\
(I-P) \mathbf{y}=(I-P) D \gamma_{n} \mathbf{u}
\end{gathered}
$$

where $\mathbf{f}=P \mathbf{f}_{0}$.

From the argument in [18], we can rewrite the cost functional as following

$$
J(\mathbf{y}, \mathbf{u})=\frac{1}{2} \int_{0}^{T} \int_{\Omega}|P \mathbf{y}|^{2} d \mathbf{x} d t+\frac{1}{2} \int_{0}^{T} \int_{\Gamma}\left(\left|R_{A}^{1 / 2} \gamma_{n} \mathbf{u}\right|^{2}+\left|\gamma_{\tau} \mathbf{u}\right|^{2}\right) d \mathbf{x} d t
$$

The control problem $\left(P_{0}\right)$ is equivalent to

$$
(P) \quad \inf \left\{J(\mathbf{y}, \mathbf{u}) \mid(\mathbf{y}, \mathbf{u}) \operatorname{satisfies}(1.6), \mathbf{u} \in V^{0,0}\left(\Sigma_{T}\right)\right\}
$$




\section{Existence of optimal solution and maximum principle}

A pair $(\mathbf{y}, \mathbf{u}) \in L^{2}\left(0, T ; V^{0}(\Omega)\right) \times L^{2}\left(0, T ; V^{0}(\Gamma)\right)$ is called an admissible pair of optimal control problem(OCP) $(\mathrm{P})$ if it is a solution to (1.6) and (1.7). An optimal solution is one admissible pair which minimizes the cost functional $J(\mathbf{y}, \mathbf{u})$.

The existence of periodic solution to Eq. (1.1) does not hold in general, and even the existence of admissible pair for the optimal control problem is not trivial when 1 is an eigenvalue of the operator $e^{-\mathcal{A} T}$.

If 1 is an eigenvalue of the operator $e^{-\mathcal{A} T}$, we denote by $\left\{\Psi_{i}\right\}_{i=1}^{N},\left\{\Psi_{i}^{*}\right\}_{i=1}^{N}$ the (normalized) linearly independent eigenfunctions corresponding to eigenvalue 1 of $e^{-\mathcal{A} T}$ and $e^{-\mathcal{A}^{*} T}$ respectively. To get the existence of admissible pair, we need the following property for operator $e^{-\mathcal{A} T}$

(UC): The solution to the equation

$$
\begin{cases}\frac{d \mathbf{z}(t)}{d t}-\nu \Delta \mathbf{z}-\left(\mathbf{f}_{1} \cdot \nabla\right) \mathbf{z}+\mathbf{z} \cdot\left(\nabla \mathbf{f}_{2}\right)^{T}+\nabla p=0, & \text { in }(0, T) \times \Omega, \\ \mathbf{z}(T)=\mathbf{z}(0), & \text { in } \Omega \\ \nabla \cdot \mathbf{z}=0, & \text { in }(0, T) \times \Omega \\ \left.\mathbf{z}\right|_{\Gamma}=0, & \end{cases}
$$

is 0 when $-\nu \frac{\partial \mathbf{z}}{\partial \mathbf{n}}+\psi \mathbf{n}-c(\psi) \mathbf{n}=0$ on $(0, T) \times \Gamma$. Here $\nabla \psi=(I-P)(\nu \triangle \mathbf{z}+$ $\left.\left(\mathbf{f}_{\mathbf{1}} \cdot \nabla\right) \mathbf{z}-\left(\nabla \mathbf{f}_{\mathbf{2}}\right)^{T} \mathbf{z}\right)$.

It is kind of unique continuation property of operator $\mathcal{A}^{*}$. It is used critically in one spot: claiming that $\left\{F^{*}\left(e^{-\mathcal{A}^{*}(T-t)} \Psi_{i}^{*}\right)\right\}_{i=1}^{N}$ are linear independent in $L^{2}\left(0, T ;\left(L^{2}(\Gamma)\right)^{2}\right)$. We can see from the definition of $\psi$ that $\psi=p$ in $H^{1}(\Omega) / \mathbb{R}$. As discussed in $[7,8]$, the unique continuation property holds for the Stokes equation. For other literatures concerned with the continuation property of Stokes-Oseen operator, we cite here [5, 6,9].

Following the similar arguments as in [21], we can proof the existence of admissible control for optimal control problem $(\mathrm{P})$.

Theorem 2.1. There exists a unique optimal solution $\left(\mathbf{y}^{*}, \mathbf{u}^{*}\right)$ for optimal control problem $(P)$ under assumptions $(H)$.

Proof. First we claim that there exists at least one admissible pair.

Indeed, from Eq. (1.6), we have that

$$
P \mathbf{y}(t)=e^{-\mathcal{A} t} P \mathbf{y}(0)+\int_{0}^{t} e^{-\mathcal{A}(t-s)}\left(\mathcal{A}_{k} P D \mathbf{u}(s)+\mathbf{f}(s)\right) d s .
$$

To get a periodic solution to Eq. (2.2), it is enough to find $\mathbf{z}_{0} \in H, \mathbf{u} \in$ $L^{2}(0, T ; U)$, such that

$$
\left(I-e^{-\mathcal{A} T}\right) \mathbf{z}_{0}=\int_{0}^{T} e^{-\mathcal{A}(T-s)}(F \mathbf{u}(s)+\mathbf{f}(s)) d s .
$$

Since the operator $e^{-\mathcal{A} T}$ is a compact operator on $H$, it follows by Fredholm alternative theorem that this trivially holds if $1 \bar{\in} \sigma\left(e^{-\mathcal{A} T}\right)$, where $\sigma\left(e^{-\mathcal{A} T}\right)$ denotes the set of the spectrum of the operator $e^{-\mathcal{A} T}$. Assume now that 


$$
1 \in \sigma\left(e^{-\mathcal{A} T}\right) .
$$

For existence of a pair $\left(\mathbf{z}_{0}, \mathbf{u}\right)$, it is suffices to prove in this case that $\forall \Psi \in \mathscr{N}=\left\{\mathbf{w} \in H ;\left(I-e^{-\mathcal{A}^{*} T}\right) \mathbf{w}=0\right\}$, the following equation holds

$$
\left\langle\int_{0}^{T} e^{-\mathcal{A}(T-s)}(F \mathbf{u}(s)+\mathbf{f}(s)) d s, \Psi\right\rangle=0 .
$$

In fact, the space $\mathscr{N}$ is finite dimensional, and $\mathscr{N}=\operatorname{span}\left\{\Psi_{i}^{*}\right\}_{i=1}^{N}$. Therefore, equation (2.4) is equivalent to

$$
\int_{0}^{T}\left\langle\mathbf{u}(s), F^{*} \boldsymbol{\Phi}_{i}(s)\right\rangle d s=\int_{0}^{T}\left\langle\mathbf{f}(s), \Phi_{i}(s)\right\rangle d s, \quad 1 \leq i \leq N,
$$

where $\Phi_{i}(s)=e^{-\mathcal{A}^{*}(T-s)} \Psi_{i}^{*}$. Inasmuch as $\Phi_{i}(s) \in D(\mathcal{A})$ a.e.s $\left.\in\right] 0, T[$, we have that $F^{*} \Phi_{i}(s)=-\nu \frac{\partial \Phi_{i}}{\partial \mathbf{n}}+\psi \mathbf{n}-c(\psi) \mathbf{n}$, where $\nabla \psi=(I-P)\left(\nu \triangle \Phi_{i}+\left(\mathbf{f}_{\mathbf{1}} \cdot \nabla\right) \Phi_{i}-\right.$ $\left.\left(\nabla \mathbf{f}_{\mathbf{2}}\right)^{T} \Phi_{i}\right)$. Taking the finite dimensional controller

$$
\mathbf{u}(t)=\sum_{j=1}^{N} c_{j} F^{*} \Phi_{j}(t)
$$

Equation (2.5) is equivalent to the following linear algebraic equation

$$
\int_{0}^{T}\left\langle\sum_{j=1}^{N} c_{j} F^{*} \Phi_{j}(s), F^{*} \Phi_{i}(s)\right\rangle_{U} d s=\int_{0}^{T}\left\langle\mathbf{f}(s), \Phi_{i}(s)\right\rangle d s, 1 \leq i \leq N,
$$

With the assumptions $(\mathrm{H})$, we can prove that $\left\{F^{*} \Phi_{i}(s)\right\}_{1 \leq i \leq N}$ are linear independent in $L^{2}(0, T ; U)$ (following the same arguments as in the proof of $(3.6 .20 \mathrm{~A})$ in $[1$, p. 55]). It follows that the matrix

$$
\left\{\int_{0}^{T}\left\langle F^{*} \Phi_{j}(s), F^{*} \Phi_{i}(s)\right\rangle_{U} d s\right\} 1 \leq i, j \leq N
$$

is full rank, and so there is $\left\{c_{j}\right\}$, or, to say, a finite dimensional controller $\mathbf{u} \in L^{2}(0, T ; U)$ such that Eq. $(2.7)$ holds. It follows that there exists a finite dimensional controller such that Eq. (1.6) has at least one periodic solution. [21]).

The existence of optimal solution of $(\mathrm{P})$ follows by a standard way (see

To get the necessary condition of $\left(\mathbf{y}^{*}, \mathbf{u}^{*}\right)$, we consider first the following approximating problem

$$
\begin{aligned}
\left(P_{\varepsilon}\right) \operatorname{Min} & \left\{\frac{1}{2} \int_{0}^{T}\left(|P \mathbf{y}|^{2}+\left|P \mathbf{y}-P \mathbf{y}^{*}\right|+\frac{1}{\varepsilon}|\mathbf{v}|^{2}\right) d \mathbf{x} d t\right. \\
+ & \left.\frac{1}{2} \int_{0}^{T}\left(\left|\mathbf{u}-\mathbf{u}^{*}\right|^{2}+\left|R_{A}^{1 / 2} \gamma_{n} \mathbf{u}\right|^{2}+\left|\gamma_{\tau} \mathbf{u}\right|^{2}\right) d \mathbf{x} d t\right\}
\end{aligned}
$$

subject to the equation

$$
P \mathbf{y}^{\prime}+\mathcal{A} P \mathbf{y}=F \mathbf{u}+\mathbf{f}+\mathbf{v}, \mathbf{y}(0)=\mathbf{y}(T) .
$$


The cost functional of problem $\left(P_{\varepsilon}\right)$ will be denoted by $J_{\varepsilon}(\cdot, \cdot)$. Problem $\left(P_{\varepsilon}\right)$ has solution $\left(\mathbf{y}_{\varepsilon}, \mathbf{u}_{\varepsilon}, \mathbf{v}_{\varepsilon}\right)$, and (see [21])

$$
\left\{\begin{array}{l}
P \mathbf{y}_{\varepsilon} \rightarrow P \mathbf{y}^{*} \text { strongly in } L^{2}\left(0, T ; V^{0}(\Omega)\right), \\
\mathbf{u}_{\varepsilon} \rightarrow \mathbf{u}^{*} \text { strongly in } L^{2}\left(0, T ; V^{0}(\Gamma)\right) \\
\mathbf{v}_{\varepsilon} \rightarrow 0 \text { weakly in } L^{2}\left(0, T ; V_{n}^{0}(\Omega)\right)
\end{array}\right.
$$

Then we can begin to derive the necessary condition for optimal solution $\left(\mathbf{y}^{*}, \mathbf{u}^{*}\right)$, i.e. the maximum principle. In fact, we shall prove that this necessary condition is also sufficient.

Theorem 2.2. The admissible pair $\left(\mathbf{y}^{*}, \mathbf{u}^{*}\right)$ is optimal solution to $O C P(P)$ if and only if there is function $\mathbf{q} \in L^{2}(0, T ; D(A)) \cap H^{1}\left([0, T], V_{n}^{0}(\Omega)\right)$ satisfying together with $\left(\mathbf{y}^{*}, \mathbf{u}^{*}\right)$ the following system

$$
\begin{cases}\frac{d \mathbf{q}}{d t}(t)-\mathcal{A}^{*} \mathbf{q}(t)=P \mathbf{y}^{*}, & t \in(0, T), \\ \mathbf{q}(0)=\mathbf{q}(T), & \\ \mathbf{u}^{*}(t)=F_{\tau}^{*} \mathbf{q}(t)+R_{A}^{-1} F_{n}^{*} \mathbf{q}(t), & \text { a.e.t } \in(0, T) .\end{cases}
$$

More precisely, we have $\mathbf{q}(t)=\mathbf{q}_{1}(t)+\mathbf{q}_{2}(t)$, where $\mathbf{q}_{1}, \mathbf{q}_{2} \in L^{2}(0, T$; $D(A)) \cap H^{1}\left([0, T], V_{n}^{0}(\Omega)\right)$ and $\mathbf{q}_{1}, \mathbf{q}_{2}$ satisfy the following equation

$$
\begin{cases}\frac{d \mathbf{q}_{1}}{d t}(t)-\mathcal{A}^{*} \mathbf{q}_{1}(t)=P \mathbf{y}^{*}(t), & t \in(0, T), \\ \frac{d \mathbf{q}_{2}}{d t}(t)-\mathcal{A}^{*} \mathbf{q}_{2}(t)=0, & t \in(0, T), \\ \mathbf{q}(0)=\mathbf{q}(T), & \\ \mathbf{u}^{*}(t)=F_{\tau}^{*} \mathbf{q}(t)+R_{A}^{-1} F_{n}^{*} q(t), & \text { a.e.t } \in(0, T) .\end{cases}
$$

Moreover, we can obtain the following regularity properties for the optimal solution via the Euler-Lagrange system.

Theorem 2.3. Let $\mathbf{f} \in L^{2}\left(0, T ;\left(V^{2 \varepsilon}(\Omega)\right)^{\prime}\right)$, then the optimal solution $\left(\mathbf{y}^{*}, \mathbf{u}^{*}\right)$ satisfies $\mathbf{u}^{*} \in V^{1-\varepsilon, 1 / 2-\varepsilon}\left(\Sigma_{T}\right), P \mathbf{y}^{*} \in V^{3 / 2-\varepsilon, 3 / 4-\varepsilon / 2}\left(Q_{T}\right)$, and the following estimate holds

$$
\left\|P \mathbf{y}^{*}\right\|_{V^{3 / 2-\varepsilon, 3 / 4-\varepsilon / 2}\left(Q_{T}\right)} \leq C\|\mathbf{f}\|_{L^{2}\left(0, T ;\left(V^{2 \varepsilon}(\Omega)\right)^{\prime}\right)} .
$$

To prove the maximum principle, we give first the following lemma about the properties of operator $\mathscr{A}: D(\mathscr{A}) \rightarrow L^{2}\left(0, T ; V_{n}^{0}(\Omega)\right)$, which is defined by

$$
\mathscr{A} \mathbf{z}=\partial_{t} \mathbf{z}+\mathcal{A} \mathbf{z}
$$

with $D(\mathscr{A})=\left\{\mathbf{z} \in L^{2}(0, T ; D(A)) \cap W^{1,2}\left([0, T] ; V_{n}^{0}(\Omega)\right) ; \mathbf{z}(0)=\mathbf{z}(T)\right\}$.

Lemma 2.4. (See Barbu [2]) The operators $\mathscr{A}, \mathscr{A}^{*}$ are closed, densely defined and have closed ranges in $L^{2}\left(0, T ; V_{n}^{0}(\Omega)\right)$. Moreover, $\operatorname{dim} N(\mathscr{A})$ and $\operatorname{dim}$ $N\left(\mathscr{A}^{*}\right)$ are finite, where $\mathscr{A}^{*}$ is the adjoint operator of $\mathscr{A}$, and the following estimates hold

$$
\begin{array}{cl}
\left\|\mathscr{A}^{-1} \mathbf{g}\right\|_{L^{2}(0, T ; D(A)) \cap W^{1,2}\left([0, T] ; V_{n}^{0}(\Omega)\right)} \leq C\|\mathbf{g}\|_{L^{2}\left(0, T ; V_{n}^{0}(\Omega)\right)}, & \forall \mathbf{g} \in R(\mathscr{A}) \\
\left\|\left(\mathscr{A}^{*}\right)^{-1} \mathbf{g}\right\|_{L^{2}(0, T ; D(A)) \cap W^{1,2}\left([0, T] ; V_{n}^{0}(\Omega)\right)} \leq C\|\mathbf{g}\|_{L^{2}\left(0, T ; V_{n}^{0}(\Omega)\right)}, & \forall \mathbf{g} \in R\left(\mathscr{A}^{*}\right) .
\end{array}
$$


Here, we have used the symbols $N$ and $R$ to denote the null space and the range of the corresponding operators.

Proof of Theorem 2.2. Step 1: Necessary condition for $\left(\mathbf{y}_{\varepsilon}, \mathbf{u}_{\varepsilon}, \mathbf{v}_{\varepsilon}\right)$. Firstly we do the following perturbation

$$
\begin{aligned}
\mathbf{u}_{\varepsilon}^{\lambda} & =\mathbf{u}_{\varepsilon}+\lambda \mathbf{u}^{0}, \\
P \mathbf{y}_{\varepsilon}^{\lambda} & =P \mathbf{y}_{\varepsilon}+\lambda\left(\mathbf{w}+P D \mathbf{u}^{0}\right), \\
\mathbf{v}_{\varepsilon}^{\lambda} & =\frac{\partial \mathbf{y}_{\varepsilon}^{\lambda}}{\partial t}+\mathcal{A} \mathbf{y}_{\varepsilon}^{\lambda}-F \mathbf{u}_{\varepsilon}^{\lambda} \\
& =\mathbf{v}_{\varepsilon}+\lambda\left(\frac{\partial \mathbf{w}}{\partial t}+\mathcal{A} \mathbf{w}+\partial_{t}\left(P D \mathbf{u}^{0}\right)-k P D \mathbf{u}^{0}\right) .
\end{aligned}
$$

where $\mathbf{w} \in D(\mathscr{A}), \mathbf{u}^{0} \in \mathscr{U}=\left\{u \in W^{1,2}\left([0, T] ; V^{0}(\Gamma)\right) ; u(0)=u(T)\right\}$. Then we get that

$$
\begin{aligned}
& \int_{0}^{T}\left(\left\langle 2 P y_{\varepsilon}-P \mathbf{y}^{*}, \mathbf{w}+P D \mathbf{u}^{0}\right\rangle+\left\langle\mathbf{u}_{\varepsilon}-\mathbf{u}^{*}, \mathbf{u}^{0}\right\rangle_{U}+\left\langle R_{A} \gamma_{n} \mathbf{u}_{\varepsilon}, \gamma_{n} \mathbf{u}^{0}\right\rangle_{U}\right. \\
& \left.\quad+\left\langle\gamma_{\tau} \mathbf{u}_{\varepsilon}, \gamma_{\tau} \mathbf{u}^{0}\right\rangle_{U}+\left\langle\frac{1}{\varepsilon} \mathbf{v}_{\varepsilon}, \frac{\partial \mathbf{w}}{\partial t}+\mathcal{A} \mathbf{w}+\partial_{t}\left(P D \mathbf{u}^{0}\right)-k P D \mathbf{u}^{0}\right\rangle\right) d t=0 .
\end{aligned}
$$

Let $\mathbf{u}^{0}=0$, the we have that

$$
\mathscr{A}^{*} \mathbf{q}_{\varepsilon}=-P\left(2 \mathbf{y}_{\varepsilon}-\mathbf{y}^{*}\right)
$$

where $\mathbf{q}_{\varepsilon}=\frac{1}{\varepsilon} \mathbf{v}_{\varepsilon}$. Moreover, we can apply (2.14) again to obtain that

$$
\begin{aligned}
& \int_{0}^{T}\left(\left\langle-F^{*} \mathbf{q}_{\varepsilon}, \mathbf{u}^{0}\right\rangle_{U}+\left\langle\mathbf{u}_{\varepsilon}-\mathbf{u}^{*}, \mathbf{u}^{0}\right\rangle_{U}+\left\langle R_{A} \gamma_{n} \mathbf{u}_{\varepsilon}, \gamma_{n} \mathbf{u}^{0}\right\rangle_{U}\right. \\
& \left.\quad+\left\langle\gamma_{\tau} \mathbf{u}_{\varepsilon}, \gamma_{\tau} \mathbf{u}^{0}\right\rangle_{U}\right) d t=0 .
\end{aligned}
$$

Hence

$$
\left\{\begin{array}{l}
-F_{\tau}^{*} \mathbf{q}_{\varepsilon}+2 \gamma_{\tau} \mathbf{u}_{\varepsilon}-\gamma_{\tau} \mathbf{u}^{*}=0, \\
-F_{n}^{*} \mathbf{q}_{\varepsilon}+\gamma_{n} \mathbf{u}_{\varepsilon}-\gamma_{\tau} \mathbf{u}^{*}+R_{A} \gamma_{n} \mathbf{u}_{\varepsilon}=0, \quad \text { a.e.t } \in(0, T) .
\end{array}\right.
$$

Step 2: Pass to limit. Decompositing $\mathbf{q}_{\varepsilon}$ to be

$$
\mathbf{q}_{\varepsilon}=\mathbf{q}_{\varepsilon}^{1}+\mathbf{q}_{\varepsilon}^{2}, \mathbf{q}_{\varepsilon}^{1} \in R(\mathscr{A}), \mathbf{q}_{\varepsilon}^{2} \in N\left(\mathscr{A}^{*}\right) .
$$

It follows from Lemma 2.4 and (2.15) that

$$
\left\|\mathbf{q}_{\varepsilon}^{1}\right\|_{L^{2}(0, T ; D(A)) \cap W^{1,2}\left([0, T] ; V_{n}^{0}(\Omega)\right)} \leq C .
$$

Because the restriction of $F$ to $N\left(\mathscr{A}^{*}\right)$ has closed range, we can decomposite $\mathbf{q}_{\varepsilon}^{2}$ to be

$$
\mathbf{q}_{\varepsilon}^{2}=\mathbf{q}_{\varepsilon}^{3}+\mathbf{q}_{\varepsilon}^{4}, \mathbf{q}_{\varepsilon}^{3} \in R(F), \mathbf{q}_{\varepsilon}^{4} \in N\left(F^{*}\right) .
$$

We can obtain from (2.17) that

$$
\left\|F^{*} \mathbf{q}_{\varepsilon}\right\|_{L^{2}\left(0, T ; V_{n}^{0}(\Omega)\right)} \leq C .
$$


It follows from (2.18) and (2.19) that

$$
\left\{\begin{array}{l}
\mathbf{q}_{\varepsilon}^{1} \rightarrow \mathbf{q}^{1} \text { weakly in } L^{2}(0, T ; D(A)) \cap W^{1,2}\left([0, T] ; V_{n}^{0}(\Omega)\right), \\
\left.\mathbf{q}_{\varepsilon}^{3} \rightarrow \mathbf{q}^{2} \text { strongly in } L^{2}(0, T ; D(A)) \cap W^{1,2}\left([0, T] ; V_{n}^{0}(\Omega)\right)\right) .
\end{array}\right.
$$

because $\mathbf{q}_{\varepsilon}^{3} \subset N\left(\mathscr{A}^{*}\right)$ and $\operatorname{dim} N\left(\mathscr{A}^{*}\right) \leq n_{0}$. Now letting $\varepsilon$ tend to 0 in $(2.15)$ and (2.17), it follows by Lemma 2.4 and (2.20) that

$$
\mathscr{A}^{*}\left(\mathbf{q}^{1}+\mathbf{q}^{2}\right)=-P \mathbf{y}^{*}, u^{*}=\left(F_{\tau}^{*}+R_{A}^{-1} F_{n}^{*}\right)\left(\mathbf{q}^{1}+\mathbf{q}^{2}\right) .
$$

Hence $\mathbf{q}=\mathbf{q}^{1}+\mathbf{q}^{2}$ satisfies Eq. (2.10). This completes the first part of proof of Theorem 2.2.

Now we prove via the class way that the solution to system (2.4) is the optimal solution to $\mathrm{OCP}(\mathrm{P})$. Letting $(\mathbf{y}, \mathbf{u})$ be arbitrary admissible pair, we have that

$$
\begin{aligned}
J\left(\mathbf{y}^{*}, \mathbf{u}^{*}\right)-J(\mathbf{y}, \mathbf{u}) \leq & \int_{0}^{T}\left\langle\frac{d \mathbf{q}}{d t}(t)-\mathcal{A}^{*} \mathbf{q}(t), P \mathbf{y}^{*}-P \mathbf{y}\right\rangle d t \\
& +\int_{0}^{T}\left(\left\langle R_{A} \gamma_{n} \mathbf{u}^{*}, \gamma_{n}\left(\mathbf{u}^{*}-\mathbf{u}\right)\right\rangle+\left\langle\gamma_{\tau} \mathbf{u}^{*}, \gamma_{\tau}\left(\mathbf{u}^{*}-\mathbf{u}\right)\right\rangle\right) d t \\
= & \int_{0}^{T}\left\langle F^{*} \mathbf{q}(t), \mathbf{u}^{*}(t)-\mathbf{u}(t)\right\rangle d t \\
& +\int_{0}^{T}\left(\left\langle R_{A} \gamma_{n} \mathbf{u}^{*}, \gamma_{n}\left(\mathbf{u}^{*}-\mathbf{u}\right)\right\rangle+\left\langle\gamma_{\tau} \mathbf{u}^{*}, \gamma_{\tau}\left(\mathbf{u}^{*}-\mathbf{u}\right)\right\rangle\right) d t \\
= & 0 .
\end{aligned}
$$

Hence, $\left(\mathbf{y}^{*}, \mathbf{u}^{*}\right)$ optimal solution $(\mathrm{P})$.

Proof of Theorem 2.3 As seen in the proof of Theorem 3.1 in [21], if 1 is not eigenvalue of operator $e^{-\mathcal{A}^{*} T}$, the pair

$$
\mathbf{u}_{1}=0, P \mathbf{y}_{1}=e^{-\mathcal{A} t}\left(I-e^{-\mathcal{A} T}\right)^{-1} \int_{0}^{T} e^{-\mathcal{A}(T-t)} \mathbf{f}(t) d t+\int_{0}^{t} e^{-\mathcal{A}(t-s)} \mathbf{f}(s) d s
$$

is an admissible pair of $\mathrm{OCP}(\mathrm{P})$. Since $\left(\mathbf{y}^{*}, \mathbf{u}^{*}\right)$ minimize the cost function $J(\mathbf{y}, \mathbf{u})$, we know that

$$
\int_{0}^{T}\left(\left|P \mathbf{y}^{*}\right|^{2}+\left|\mathbf{u}^{*}\right|^{2}\right) \leq \int_{0}^{T}\left|P \mathbf{y}_{1}\right|^{2} d t \leq C\|\mathbf{f}\|_{L^{2}\left(0, T ;\left(V^{2 \varepsilon}(\Omega)\right)^{\prime}\right)}^{2} .
$$

If 1 is eigenvalue of operator $e^{-\mathcal{A}^{*} T}$, we can take a finite dimensional input $\mathbf{u}_{2}(t, \mathbf{x})=\sum_{j=1}^{N} c_{j} \frac{\partial}{\partial \mathbf{n}} e^{-\mathcal{A}^{*}(T-t)} \Psi_{j}^{*}$, where $\left\{\Psi_{j}^{*}\right\}_{1 \leq j \leq N}$ are the eigenfunctions of operator $e^{-\mathcal{A}^{*} T}$ corresponding to eigenvalue 1 . As seen in the proof of Theorem 3.1 in [21], we know that $\left\{c_{j}\right\}_{1 \leq j \leq N}$ is the solution to one algebraic equation, and we have

$$
\sum_{i=1}^{i=N}\left|c_{i}\right|^{2} \leq C\|\mathbf{f}\|_{L^{2}\left(0, T ;\left(V^{2 \varepsilon}(\Omega)\right)^{\prime}\right)}^{2} .
$$

Moreover, we can find $P \mathbf{y}_{2}(0)=P \mathbf{y}_{2}(T) \in V_{n}^{0}(\Omega)$ satisfying

$$
\left|P \mathbf{y}_{2}(0)\right| \leq C\|\mathbf{f}\|_{L^{2}\left(0, T ;\left(V^{2 \varepsilon}(\Omega)\right)^{\prime}\right)} \text {. }
$$


Hence

$$
\int_{0}^{T}\left(\left|P \mathbf{y}^{*}\right|^{2}+\left|\mathbf{u}^{*}\right|^{2}\right) \leq \int_{0}^{T}\left|P \mathbf{y}_{2}\right|^{2}+\left|\mathbf{u}_{2}\right|^{2} d t \leq C\|\mathbf{f}\|_{L^{2}\left(0, T ;\left(V^{2 \varepsilon}(\Omega)\right)^{\prime}\right)}^{2} .
$$

Letting $\mathbf{w}$ be the solution to the equation

$$
\left\{\begin{array}{l}
\partial_{t} \mathbf{w}+\mathcal{A}_{k} \mathbf{w}=\mathcal{A}_{k} P D \mathbf{u}^{*} \\
\mathbf{w}(0)=\mathbf{w}(T)
\end{array}\right.
$$

we see that $\mathbf{z}=P \mathbf{y}^{*}-\mathbf{w}$ is the solution to the equation

$$
\left\{\begin{array}{l}
\partial_{t} \mathbf{z}+\mathcal{A}_{k} \mathbf{z}=k P \mathbf{y}^{*}+\mathbf{f}, \\
\mathbf{z}(0)=\mathbf{z}(T)
\end{array}\right.
$$

From Appendix Theorem 4.2, we know that

$$
\mathbf{w} \in L^{2}\left(0, T ; D\left(\mathcal{A}^{1 / 4-\varepsilon}\right)\right) \cap H^{1 / 4-\varepsilon}\left(0, T ; V^{0}(\Omega)\right) .
$$

Since $P \mathbf{y}^{*} \in L^{2}\left(0, T ; V_{n}^{0}(\Omega)\right)$, it follows that

$$
\mathbf{z} \in L^{2}\left(0, T ; D\left(\mathcal{A}^{1 / 4-\varepsilon}\right)\right) \cap H^{1 / 4-\varepsilon}\left(0, T ; V^{0}(\Omega)\right) .
$$

So, $P \mathbf{y}^{*} \in L^{2}\left(0, T ; D\left(\mathcal{A}^{1 / 4-\varepsilon}\right)\right) \cap H^{1 / 4-\varepsilon}\left(0, T ; V^{0}(\Omega)\right)$, moreover,

$$
\left\|P \mathbf{y}^{*}\right\|_{L^{2}\left(0, T ; D\left(\mathcal{A}^{1 / 4-\varepsilon}\right)\right) \cap H^{1 / 4-\varepsilon}\left(0, T ; V^{0}(\Omega)\right)} \leq C\|\mathbf{f}\|_{L^{2}\left(0, T ;\left(V^{2 \varepsilon}(\Omega)\right)^{\prime}\right)} .
$$

Considering now $\phi$ as the solution to the equation

$$
\left\{\begin{array}{l}
\partial_{t} \phi-\mathcal{A}_{k}^{*} \phi=-P \mathbf{y}^{*}, \\
\phi(0)=\phi(T)
\end{array}\right.
$$

We see that $\psi=\mathbf{q}-\phi$ is the solution to the equation

$$
\left\{\begin{array}{l}
\partial_{t} \psi-\mathcal{A}_{k}^{*} \psi=-k \mathbf{q} \\
\phi(0)=\phi(T)
\end{array}\right.
$$

As seen in the proof of Theorem 2.2, $\mathbf{q}=\mathbf{q}_{1}+\mathbf{q}_{2}$, and

$$
\begin{gathered}
\left\|\mathbf{q}_{1}\right\|_{L^{2}(0, T ; D(A)) \cap W^{1,2}\left([0, T] ; V_{n}^{0}(\Omega)\right)} \leq C\left\|P \mathbf{y}^{*}\right\|_{L^{2}\left(0, T ; V_{n}^{0}(\Omega)\right)}, \\
\left\|\mathbf{q}_{2}\right\|_{L^{2}(0, T ; D(A)) \cap W^{1,2}\left([0, T] ; V_{n}^{0}(\Omega)\right)} \leq C\left\|\mathbf{u}^{*}\right\|_{L^{2}\left(0, T ; V_{n}^{0}(\Gamma)\right)} .
\end{gathered}
$$

It follows from (2.21) and (2.23) that

$$
\|\mathbf{q}\|_{L^{2}(0, T ; D(A)) \cap W^{1,2}\left([0, T] ; V_{n}^{0}(\Omega)\right)} \leq C\|\mathbf{f}\|_{L^{2}\left(0, T ;\left(V^{2 \varepsilon}(\Omega)\right)^{\prime}\right)} .
$$

By Appendix Theorem 4.4, we know that $\frac{\partial \phi}{\partial \mathbf{n}} \in V^{1 / 2-\varepsilon, 1 / 4-\varepsilon / 2}\left(Q_{T}\right)$, and so $\frac{\partial \mathbf{q}}{\partial \mathbf{n}} \in V^{1 / 2-\varepsilon, 1 / 4-\varepsilon / 2}\left(Q_{T}\right)$, moreover,

$$
\left\|\frac{\partial \mathbf{q}}{\partial \mathbf{n}}\right\|_{V^{1 / 2-\varepsilon, 1 / 4-\varepsilon / 2}\left(Q_{T}\right)} \leq C\|\mathbf{f}\|_{L^{2}\left(0, T ;\left(V^{2 \varepsilon}(\Omega)\right)^{\prime}\right)} .
$$

It follows from Proposition 1.4 that $\mathbf{u}^{*} \in V^{1 / 2-\varepsilon, 1 / 4-\varepsilon / 2}\left(\Sigma_{T}\right)$. Applying again Theorem 4.2 we get by the same arguments that $P \mathbf{y}^{*} \in V^{1-\varepsilon, 1 / 2-\varepsilon}\left(Q_{T}\right)$, and repeating the same analysis as above, we see that $\mathbf{u}^{*} \in V^{1-\varepsilon, 1 / 2-\varepsilon / 2}\left(\Sigma_{T}\right)$. It follows that $P \mathbf{y}^{*} \in V^{3 / 2-\varepsilon, 3 / 4-\varepsilon}\left(Q_{T}\right)$ by Theorem 4.2. From above analysis, we have moreover that estimate $(2.12)$ holds. In particular, it follows by interpolation theory that $\mathbf{q} \in C\left([0, T] ; V^{2}(\Omega) \cap V_{0}^{1}(\Omega)\right)$, and $P \mathbf{y}^{*} \in C\left([0, T] ; V_{n}^{0}(\Omega)\right)$. 


\section{Feedback control and application to periodic Navier-Stokes equations}

In the sequel we shall only consider the tangential Dirichlet boundary control for the convenient to present the idea of the feedback synthesis and it's application. Denote here by $H$ the space $V_{n}^{0}(\Omega)$.

To get the optimal feedback controller of optimal control problem $(\mathrm{P})$, we consider the dual optimal control problem:

$$
\begin{gathered}
\min \left\{\int_{0}^{T}\left(\frac{1}{2}\left(\left|F^{*} \mathbf{p}(t)\right|^{2}+|\mathbf{y}(t)|^{2}\right)+\langle\mathbf{f}(t), \mathbf{p}(t)\rangle\right) d t\right. \\
\left.\mathbf{p}^{\prime}-\mathcal{A}^{*} \mathbf{p}=\mathbf{y}, \mathbf{p}(0)=\mathbf{p}(T), \mathbf{y} \in L^{2}(0, T ; H)\right\} .
\end{gathered}
$$

Equivalently,

$$
\begin{gathered}
\min \left\{\int_{0}^{T}\left(\frac{1}{2}\left(\left|F^{*} \mathbf{p}(t)\right|^{2}+|\mathbf{y}(t)|^{2}\right)+\langle\mathbf{f}(T-t), \mathbf{p}(t)\rangle\right) d t\right. \\
\left.\mathbf{p}^{\prime}+\mathcal{A}^{*} \mathbf{p}=\mathbf{y}, \mathbf{p}(0)=\mathbf{p}(T), \mathbf{y} \in L^{2}(0, T ; H)\right\} .
\end{gathered}
$$

If $(\mathbf{p}, \mathbf{y})$ is an optimal pair in problem $(3.1)$, then it follows via maximum principle and duality arguments that $\left(-\mathbf{y}(T-t),-F^{*} \mathbf{p}(T-t)\right)$ is optimal in problem $(\mathrm{P})$.

The dynamic programming equation corresponding to problem (3.2) is

$$
\psi_{t}(t, \mathbf{p})+\frac{1}{2}\left|\psi_{\mathbf{p}}(t, \mathbf{p})\right|^{2}+\left\langle\mathcal{A}^{*} \mathbf{p}, \psi_{\mathbf{p}}(t, \mathbf{p})\right\rangle=\frac{1}{2}\left|F^{*} \mathbf{p}\right|^{2}+\langle\mathbf{f}(t), \mathbf{p}\rangle,
$$

where $\psi_{\mathbf{p}}=\nabla_{p} \psi$.

For the existence of solution to system (3.3), we have the following Theorem.

Theorem 3.1. There exist a unique real number $\mu$, and a continuous function $\psi:[0, T] \times H \rightarrow \mathbb{R}$ which is convex and Gateaux differentiable in $\mathbf{p}$, absolutely continuous in $t$ for each $\mathbf{p} \in D(A)$, satisfies a.e. in $(0, T) E q .(3.3)$, and

$$
\psi(0, \mathbf{p})-\psi(T, \mathbf{p})=\mu, \quad \forall \mathbf{p} \in D(A) .
$$

Before proving Theorem 3.1, we pause briefly to present a few consequences of the above Theorem.

Consider the following closed-loop dual equation

$$
\mathbf{p}^{\prime}+\mathcal{A}^{*} \mathbf{p}+\psi_{\mathbf{p}}(T-t, \mathbf{p}(t))=0, t \in(0, T) ; \mathbf{p}(0)=\mathbf{p}(T),
$$

Since the cost functional of optimal control problem is quadratic, letting

$$
\psi(t, \mathbf{p})=\frac{1}{2}\langle Q \mathbf{p}, \mathbf{p}\rangle+\langle\mathbf{r}(t), \mathbf{p}\rangle+h(t),
$$


where $Q$ is positive and self-adjoint operators, $h$ is a function satisfying $h(0)=$ $h(T)+\mu$ we have by $(3.3)$ that (see $[13,16])$

$$
\left\{\begin{array}{l}
Q \mathcal{A}+\mathcal{A}^{*} Q+Q^{2}=F F^{*} \\
\mathbf{r}^{\prime}(t)+\mathcal{A} \mathbf{r}(t)+Q \mathbf{r}(t)=\mathbf{f}(t) ; \mathbf{r}(0)=\mathbf{r}(T)
\end{array}\right.
$$

We can see from above that equation (3.5) is in fact a linear periodic equation. With Theorem 5.3 and Remark 5.3 in Part V-1 of [16], we know that equation (3.5) admits a periodic solution if $\left(\mathcal{A}^{*}, F^{*}\right)$ is detectable. This can be implied by the unique continuation property of $\mathcal{A}^{*}$, which is always satisfied as we stated.

Let $p$ be a solution to Eq. (3.5). Consider the feedback law,

$$
\mathbf{z}^{*}(t)=-\psi_{\mathbf{p}}(T-t, \mathbf{p}(t)), \quad t \in(0, T),
$$

where $\mathbf{p}$ is the solution to closed loop system. If $\mathbf{p}^{*}$ is a solution to equation (3.5), then we claim that the pair $\left(\mathbf{p}^{*}(t),-\psi_{\mathbf{p}}\left(T-t, \mathbf{p}^{*}(t)\right)\right)$ is the optimal solution of the dual problem (3.2).

Indeed, for any admissible pair (q, y) of OCP (3.2), we know from (3.3) that

$$
\begin{aligned}
\frac{d}{d t} \psi & (T-t, \mathbf{q}(t)) \\
= & \left\langle\psi_{\mathbf{p}}(T-t, \mathbf{q}(t)),-\mathcal{A}^{*} \mathbf{q}(t)+\mathbf{y}(t)\right\rangle+\frac{1}{2}\left|\psi_{\mathbf{p}}(T-t, \mathbf{q}(t))\right|^{2} \\
& +\left\langle\mathcal{A}^{*} \mathbf{q}(t), \psi_{\mathbf{p}}(T-t, \mathbf{q}(t))\right\rangle-\frac{1}{2}\left|F^{*} \mathbf{q}(t)\right|^{2}-\langle\mathbf{f}(T-t), \mathbf{q}(t)\rangle \\
\geq & -\frac{1}{2}|\mathbf{y}(t)|^{2}-\frac{1}{2}\left|F^{*} \mathbf{q}(t)\right|^{2}-\langle\mathbf{f}(T-t), \mathbf{q}(t)\rangle .
\end{aligned}
$$

Integrating on $(0, T)$, it follows from (3.4) that

$$
\int_{0}^{T}\left(\frac{1}{2}\left(|\mathbf{y}(t)|^{2}+\left|F^{*} \mathbf{q}(t)\right|^{2}\right)+\langle\mathbf{f}(T-t), \mathbf{q}(t)\rangle\right) d t \geq-\mu .
$$

Moreover, we can get by the similar calculation as above that

$$
\int_{0}^{T}\left(\frac{1}{2}\left(\left|\mathbf{z}^{*}(t)\right|^{2}+\left|F^{*} \mathbf{p}^{*}(t)\right|^{2}\right)+\left\langle\mathbf{f}(T-t), \mathbf{p}^{*}(t)\right\rangle\right) d t=-\mu .
$$

From the arguments above we see that $\left(\mathbf{p}^{*}(t),-\psi_{\mathbf{p}}\left(T-t, \mathbf{p}^{*}(t)\right)\right)$ is the optimal solution of the dual problem (3.2) and $-\mu$ is the minimum of the cost functional.

As mentioned earlier, the pair

$$
\mathbf{y}^{*}(t)=\psi_{\mathbf{p}}\left(t, \mathbf{p}^{*}(T-t)\right), \mathbf{u}^{*}(t)=-F^{*} \mathbf{p}^{*}(T-t)
$$

is optimal in problem $(\mathrm{P})$. In other words,

$$
\mathbf{u}^{*}(t)=-F^{*} \psi_{\mathbf{y}}^{*}\left(t, \mathbf{y}^{*}(t)\right), \quad t \in(0, T)
$$

is an optimal feedback controller for problem $(\mathrm{P})$. Here $\psi^{*}$ is the conjugate of the function $\mathbf{p} \rightarrow \psi(t, \mathbf{p})$. Summarizing we have 
Theorem 3.2. Assume that the periodic problem (3.5) has a mild solution $\mathbf{p} \in$ $C([0, T] ; H)$. Then the feedback control $(3.11)$ is optimal in problem $(P)$.

The way to prove Theorem 3.2 is classical, and we refer to $[2,12]$.

Proof of Theorem 3.1 To get the existence of solution to the Hamilton-JacobiBellman equation, we give first the following lemma.

Lemma 3.3. There exist a continuous function $\varphi^{0}: V \rightarrow \mathbb{R}$, and a unique $\mu \in \mathbb{R}$ such that

$$
\begin{aligned}
\varphi^{0}(\mathbf{x})= & \inf \left\{\int_{0}^{T}\left(\frac{1}{2}\left(\left|F^{*} \mathbf{p}(t)\right|^{2}+|\mathbf{y}(t)|^{2}\right)+\langle\mathbf{g}(t), \mathbf{p}(t)\rangle\right) d t+\varphi^{0}(\mathbf{p}(T)) ;\right. \\
& \left.\mathbf{p}^{\prime}(t)+\mathcal{A}^{*} \mathbf{p}(t)=\mathbf{y}(t), \mathbf{p}(0)=\mathbf{x}, \mathbf{y} \in L^{2}(0, T ; H)\right\}+\mu, \quad \forall \mathbf{x} \in V .
\end{aligned}
$$

where $\mathbf{g}(t)=\mathbf{f}(T-t)$.

Proof. Step 1: Define a sequence of value function by iteration. Let $\varphi_{n}: V \rightarrow \mathbb{R}$ be defined by

$$
\begin{aligned}
\varphi_{n}(\mathbf{x})= & \inf _{\mathbf{z}}\left\{\int_{0}^{T}\left(\frac{1}{2}\left(\left|F^{*} \mathbf{q}(t)\right|^{2}+|\mathbf{z}(t)|^{2}\right)+\langle\mathbf{g}(t), \mathbf{q}(t)\rangle\right) d t+\varphi_{n-1}(\mathbf{q}(T)) ;\right. \\
& \left.\mathbf{q}^{\prime}(t)+\mathcal{A}^{*} \mathbf{q}(t)=\mathbf{z}(t), \mathbf{q}(0)=\mathbf{x}, \mathbf{z} \in L^{2}(0, T ; H)\right\}
\end{aligned}
$$

We take $\varphi_{0}(\mathbf{x})=0$. It's well known that $\varphi_{n}$ are continuous, convex and the infimum defining $\varphi_{n}(\mathbf{x})$ is attained. Moreover, since $\mathbf{g}$ is $T$ - periodic, we may equivalently write $\varphi_{n}(\mathbf{x})$ as

$$
\begin{aligned}
\varphi_{n}(\mathbf{x})= & \inf \left\{\int_{0}^{n T}\left(\frac{1}{2}\left(\left|F^{*} \mathbf{q}(t)\right|^{2}+|\mathbf{z}(t)|^{2}\right)+\langle\mathbf{g}(t), \mathbf{q}(t)\rangle\right) d t ;\right. \\
& \left.\mathbf{q}^{\prime}(t)+\mathcal{A}^{*} \mathbf{q}(t)=\mathbf{z}(t) \text { in }(0, n T), \mathbf{q}(0)=\mathbf{x}, \mathbf{z} \in L^{2}(0, n T ; H)\right\} .
\end{aligned}
$$

Let $\left(\mathbf{q}_{n}, \mathbf{z}_{n}\right)$ be an optimal pair in problem (3.14). By the maximal principle, there are $\mathbf{z}_{n} \in C\left([0, n T] ; D(A)^{\prime}\right)$ such that

$$
\left\{\begin{array}{l}
\mathbf{q}_{n}^{\prime}(t)+\mathcal{A}^{*} \mathbf{q}_{n}(t)=\mathbf{z}_{n}(t) \\
\mathbf{z}_{n}^{\prime}(t)-\mathcal{A} \mathbf{z}_{n}(t)=F F^{*} \mathbf{q}_{n}(t)+\mathbf{g} \\
\mathbf{q}_{n}(0)=\mathbf{x}, \mathbf{z}_{n}(n T)=0
\end{array}\right.
$$

We recall also that $\nabla \varphi_{n}(\mathbf{x})=-\mathbf{z}_{n}(0)$. 
Step 2: Convergence of $\left\{\nabla \varphi_{n}(\mathbf{x})\right\}$. We note first that under our assumptions there are functions $(\overline{\mathbf{q}}, \overline{\mathbf{z}})$ such that

$$
\left\{\begin{array}{l}
\overline{\mathbf{q}}^{\prime}(t)+\mathcal{A}^{*} \overline{\mathbf{q}}(t)=\overline{\mathbf{z}}(t), \quad t \in(0, n T) \\
\overline{\mathbf{z}}^{\prime}(t)-\mathcal{A} \overline{\mathbf{z}}(t)=F F^{*} \overline{\mathbf{q}}(t)+\mathbf{g}, \quad t \in(0, n T) \\
\overline{\mathbf{q}}(0)=\overline{\mathbf{q}}(T), \quad \overline{\mathbf{z}}(0)=\overline{\mathbf{z}}(T)
\end{array}\right.
$$

We extend by periodicity $(\overline{\mathbf{q}}, \overline{\mathbf{z}})$ on the time interval $(0, n T)$, and note that

$$
\left\{\begin{array}{l}
\frac{d}{d t}\left(\mathbf{q}_{n}-\overline{\mathbf{q}}\right)+\mathcal{A}^{*}\left(\mathbf{q}_{n}-\overline{\mathbf{q}}\right)=\mathbf{z}_{n}-\overline{\mathbf{z}}, \quad t \in(0, n T), \\
\frac{d}{d t}\left(\mathbf{z}_{n}-\overline{\mathbf{z}}\right)-\mathcal{A}\left(\mathbf{z}_{n}-\overline{\mathbf{z}}\right)=F F^{*}\left(\mathbf{q}_{n}-\overline{\mathbf{q}}\right), \quad t \in(0, n T), \\
\left(\mathbf{q}_{n}-\overline{\mathbf{q}}\right)(0)=\mathbf{x}-\overline{\mathbf{q}}(0), \quad\left(\mathbf{z}_{n}-\overline{\mathbf{z}}\right)(n T)=-\overline{\mathbf{z}}(0) .
\end{array}\right.
$$

As stated in Theorem 2.3, $\overline{\mathbf{q}} \in C([0, T] ; D(A)), \overline{\mathbf{z}} \in C([0, T] ; H)$. Then,

$$
\frac{d}{d t}\left\langle\mathbf{q}_{n}-\overline{\mathbf{q}}, \mathbf{z}_{n}-\overline{\mathbf{z}}\right\rangle=\left|\mathbf{z}_{n}-\overline{\mathbf{z}}\right|^{2}+\left|F^{*}\left(\mathbf{q}_{n}-\overline{\mathbf{q}}\right)\right|^{2}, \quad t \in(0, n T) .
$$

Integrating on $(0, n T)$, we get that

$$
\begin{aligned}
& \int_{0}^{n T}\left(\left|\mathbf{z}_{n}-\overline{\mathbf{z}}\right|^{2}+\left|F^{*}\left(\mathbf{q}_{n}-\overline{\mathbf{q}}\right)\right|^{2}\right) d t \\
& \quad=\left\langle\mathbf{q}_{n}(n T)-\overline{\mathbf{q}}(n T), \overline{\mathbf{z}}(0)\right\rangle-\left\langle\mathbf{x}-\overline{\mathbf{q}}(0), \mathbf{z}_{n}(0)-\overline{\mathbf{z}}(0)\right\rangle
\end{aligned}
$$

From Theorem 4.5 in [18], we know that there is operator $\Pi \in \mathscr{L}(H ; D(A))$ such that the operator

$$
A_{\Pi}: A_{\Pi \mathbf{y}}=\mathcal{A} \mathbf{y}-F F^{*} \Pi \mathbf{y}, \quad \forall \mathbf{y} \in D\left(A_{\Pi}\right)
$$

is the infinitesimal generator of an analytic exponentially stable semigroup on $H$. We can rewrite equations (3.17) as

$$
\left\{\begin{array}{l}
\frac{d}{d t}\left(\mathbf{q}_{n}-\overline{\mathbf{q}}\right)+\left(\mathcal{A}^{*}-\Pi^{*} F F^{*}\right)\left(\mathbf{q}_{n}-\overline{\mathbf{q}}\right)=\mathbf{z}_{n}-\overline{\mathbf{z}}-\Pi^{*} F F^{*}\left(\mathbf{q}_{n}-\overline{\mathbf{q}}\right), \\
\frac{d}{d t}\left(\mathbf{z}_{n}-\overline{\mathbf{z}}\right)-\mathcal{A}_{k}\left(\mathbf{z}_{n}-\overline{\mathbf{z}}\right)=F F^{*}\left(\mathbf{q}_{n}-\overline{\mathbf{q}}\right)-k\left(\mathbf{z}_{n}-\overline{\mathbf{z}}\right), \quad t \in(0, n T), \\
\left(\mathbf{q}_{n}-\overline{\mathbf{q}}\right)(0)=\mathbf{x}-\overline{\mathbf{q}}(0), \quad\left(\mathbf{z}_{n}-\overline{\mathbf{z}}\right)(n T)=-\overline{\mathbf{z}}(0) .
\end{array}\right.
$$

Then

$$
\begin{aligned}
\left(\mathbf{q}_{n}-\overline{\mathbf{q}}\right)(t)= & e^{-A_{\Pi}^{*} t}(\mathbf{x}-\overline{\mathbf{q}}(0))+\int_{0}^{t} e^{-A_{\Pi}^{*}(t-s)}\left(\left(\mathbf{z}_{n}-\overline{\mathbf{z}}\right)\right. \\
& \left.-\Pi^{*} F F^{*}\left(\mathbf{q}_{n}-\overline{\mathbf{q}}\right)\right) d s
\end{aligned}
$$

It follows that

$$
\begin{aligned}
\left|\left(\mathbf{q}_{n}-\overline{\mathbf{q}}\right)(t)\right| \leq & |\mathbf{x}-\overline{\mathbf{q}}(0)|+C\left(\left\|\left(\mathbf{z}_{n}-\overline{\mathbf{z}}\right)\right\|_{L^{2}(0, n T ; H)}\right. \\
& \left.+\left\|F^{*}\left(\mathbf{q}_{n}-\overline{\mathbf{q}}\right)\right\|_{L^{2}(0, n T ; H)}\right)
\end{aligned}
$$

Similarly,

$$
\begin{aligned}
\left(\mathbf{z}_{n}-\overline{\mathbf{z}}\right)(t)= & e^{-A_{k}^{*}(n T-t)} \overline{\mathbf{z}}(0) \\
& +\int_{t}^{n T} e^{-A_{k}^{*}(s-t)}\left(-k\left(\mathbf{z}_{n}-\overline{\mathbf{z}}\right)(s)+F F^{*}\left(\mathbf{q}_{n}-\overline{\mathbf{q}}\right)(s)\right) d s .
\end{aligned}
$$


Since

$$
\begin{aligned}
& \left|A_{k}^{1 / 2} \int_{t}^{n T} e^{-A_{k}^{*}(s-t)} F F^{*}\left(\mathbf{q}_{n}-\overline{\mathbf{q}}\right)(s) d s\right| \\
& =\left|\int_{t}^{n T} e^{-A_{k}^{*}(s-t)} A_{k}^{1 / 4+\varepsilon} A_{k}^{1 / 4-\varepsilon} D F^{*}\left(\mathbf{q}_{n}-\overline{\mathbf{q}}\right)(s) d s\right| \\
& \leq\left(\int_{t}^{n T} \frac{e^{-2 \omega(s-t)}}{(s-t)^{1 / 2+\varepsilon}}\right)^{1 / 2}\left(\int_{0}^{n T}\left|F^{*}\left(\mathbf{q}_{n}-\overline{\mathbf{q}}\right)(t)\right|^{2} d t\right)^{1 / 2} \\
& \quad \leq C \int_{0}^{n T}\left|F^{*}\left(\mathbf{q}_{n}-\overline{\mathbf{q}}\right)(s)\right|^{2} d t
\end{aligned}
$$

we obtain that

$$
\left\|\left(z_{n}-\overline{\mathbf{z}}\right)(t)\right\|_{V^{\prime}} \leq|\overline{\mathbf{z}}(0)|+C\left(\left\|\left(\mathbf{z}_{n}-\overline{\mathbf{z}}\right)\right\|_{L^{2}(0, n T ; H)}+\left\|F^{*}\left(\mathbf{q}_{n}-\overline{\mathbf{q}}\right)\right\|_{L^{2}(0, n T ; H)}\right) .
$$

Combining (3.18), (3.21), (3.23), we get that

$$
\int_{0}^{n T}\left|\left(\mathbf{z}_{n}-\overline{\mathbf{z}}\right)(t)\right|^{2} d t+\int_{0}^{n T}\left|F^{*}\left(\mathbf{q}_{n}-\overline{\mathbf{q}}\right)(t)\right|^{2} d t \leq C(\mathbf{f}, \mathbf{x}),
$$

where $C(\mathbf{f}, \mathbf{x})$ is dependent of the quantities of $\|\mathbf{f}\|_{L^{2}\left(0, T ;\left(V^{2 \varepsilon}(\Omega)\right)^{\prime}\right)}$ and $\|\mathbf{x}\|$, but independent of $n$.

Applying again (3.20) and (3.24), we see that

$$
\left\|\mathbf{q}_{n}-\overline{\mathbf{q}}\right\|_{L^{2}(0, n T ; H)} \leq C \int_{0}^{n T}\left(\left|\left(\mathbf{z}_{n}-\overline{\mathbf{z}}\right)(t)\right|^{2}+\left|F^{*}\left(\mathbf{q}_{n}-\overline{\mathbf{q}}\right)(t)\right|^{2}\right) d t \leq C .
$$

Equation (3.17) can be written as

$$
\left\{\begin{array}{l}
\frac{d}{d t}\left(\mathbf{q}_{n}-\overline{\mathbf{q}}\right)+\mathcal{A}_{k}^{*}\left(\mathbf{q}_{n}-\overline{\mathbf{q}}\right)=\mathbf{z}_{n}-\overline{\mathbf{z}}+k\left(\mathbf{q}_{n}-\overline{\mathbf{q}}\right), \quad t \in(0, n T), \\
\frac{d}{d t}\left(\mathbf{z}_{n}-\overline{\mathbf{z}}\right)-\mathcal{A}_{k}\left(\mathbf{z}_{n}-\overline{\mathbf{z}}\right)=F F^{*}\left(\mathbf{q}_{n}-\overline{\mathbf{q}}\right)-k\left(\mathbf{z}_{n}-\overline{\mathbf{z}}\right), \quad t \in(0, n T), \\
\left(\mathbf{q}_{n}-\overline{\mathbf{q}}\right)(0)=\mathbf{x}-\overline{\mathbf{q}}(0), \quad\left(\mathbf{z}_{n}-\overline{\mathbf{z}}\right)(n T)=-\overline{\mathbf{z}}(0) .
\end{array}\right.
$$

Applying the arguments in Theorem 3.6 in [18], we get that

$$
\left\|\mathbf{z}_{n}-\overline{\mathbf{z}}\right\|_{V^{1,1 / 2}\left(Q_{n T}\right)}+\left\|\mathbf{q}_{n}-\overline{\mathbf{q}}\right\|_{V^{3,3 / 2}\left(Q_{n T}\right)} \leq C .
$$

Moreover,

$$
\left\|\mathbf{z}_{n}\right\|_{D(A)^{\prime}}+\left|\mathbf{q}_{n}\right| \leq C, \quad \forall t \in(0, n T) .
$$

Hence, on a subsequence, again denoted by $n$, we have

$$
\left\{\begin{array}{l}
F^{*}\left(\mathbf{q}_{n}-\overline{\mathbf{q}}\right) \rightarrow F^{*}\left(\mathbf{q}^{*}-\overline{\mathbf{q}}\right), \text { weakly in } L^{2}\left(\mathbb{R}^{+} ; U\right), \\
\mathbf{z}_{n}-\overline{\mathbf{z}} \rightarrow \mathbf{z}^{*}-\overline{\mathbf{z}} \text { weakly in } L^{2}\left(\mathbb{R}^{+} ; H\right) \\
\mathbf{q}_{n}-\overline{\mathbf{q}} \rightarrow \mathbf{q}^{*}-\overline{\mathbf{q}} \text { weakly in } L^{2}\left(\mathbb{R}^{+} ; H\right)
\end{array}\right.
$$

Moreover, by (3.26), we see that

$$
\mathbf{q}_{n}(t) \rightarrow \mathbf{q}^{*}(t), \text { weakly in } H, \quad \forall t \geq 0,
$$


and

$$
\left(\mathbf{q}^{*}-\overline{\mathbf{q}}\right)(t)=e^{-A_{k}^{*} t}(\mathbf{x}-\overline{\mathbf{q}}(0))+\int_{0}^{t} e^{-A_{k}^{*}(t-s)}\left(\left(\mathbf{z}^{*}-\overline{\mathbf{z}}\right)(s)+k\left(\mathbf{q}^{*}-\overline{\mathbf{q}}\right)(s)\right) d s .
$$

Similarly, it follows that

$$
\mathbf{z}_{n}(t) \rightarrow \mathbf{z}^{*}(t), \text { weakly in } V^{\prime}, \quad \forall t \geq 0,
$$

and

$$
\left(\mathbf{z}^{*}-\overline{\mathbf{z}}\right)(t)=\int_{t}^{\infty} e^{-A_{k}(s-t)}\left(-k\left(\mathbf{z}^{*}-\overline{\mathbf{z}}\right)(s)+F F^{*}\left(\mathbf{q}^{*}-\overline{\mathbf{q}}\right)(s)\right) d s .
$$

In other words, $\left(\mathbf{q}^{*}, \mathbf{z}^{*}\right)$ is the weak solution to system

$$
\left\{\begin{array}{l}
\mathbf{q}^{*^{\prime}}(t)+\mathcal{A}^{*} \mathbf{q}^{*}(t)=\mathbf{z}^{*}(t) \\
\mathbf{z}^{*^{\prime}}(t)-\mathcal{A} \mathbf{z}^{*}(t)=F F^{*} \mathbf{q}^{*}(t)+\mathbf{g} \\
\mathbf{q}^{*}(0)=\mathbf{x}, \mathbf{z}^{*} \in L^{\infty}\left(\mathbb{R}^{+} ; H\right)
\end{array}\right.
$$

By Eq. (3.15), (3.32), we get that

$\int_{0}^{n T}\left(\left|\mathbf{z}_{n}-\mathbf{z}^{*}\right|^{2}+\left|F^{*}\left(\mathbf{q}_{n}-\mathbf{q}^{*}\right)\right|^{2}\right) d t=\left\langle\mathbf{q}_{n}(n T)-\mathbf{q}^{*}(n T),-\mathbf{z}^{*}(n T)\right\rangle$.

It follows by (3.31) that

$$
\lim _{t \rightarrow \infty}\left(\mathbf{z}^{*}-\mathbf{z}^{*}\right)(t)=0, \text { strongly in } V^{\prime} .
$$

We have therefore,

$$
\lim _{n \rightarrow \infty}\left(\mathbf{z}^{*}-\overline{\mathbf{z}}\right)(n T)=0, \text { strongly in } V^{\prime} .
$$

On the other hand, it follows by (3.27) and the interpolation theory (see [19]) that

$$
\begin{aligned}
& \left\|\left(\mathbf{q}_{n}-\mathbf{q}^{*}\right)(n T)\right\|_{D(A)} \\
& \quad \leq\left\|\left(\mathbf{q}_{n}-\overline{\mathbf{q}}\right)(n T)\right\|_{D(A)}+\left\|\left(\overline{\mathbf{q}}-\mathbf{q}^{*}\right)(n T)\right\|_{D(A)} \\
& \quad \leq C\left(\left\|\mathbf{q}_{n}-\overline{\mathbf{q}}\right\|_{V^{3,3 / 2}\left(Q_{n T}\right)}+\left\|\overline{\mathbf{q}}-\mathbf{q}^{*}\right\|_{V^{3,3 / 2}\left(Q_{\infty}\right)}\right) \\
& \quad \leq C
\end{aligned}
$$

where $C$ is independent of $n$. Hence,

$$
\mathbf{q}_{n}(n T)-\mathbf{q}^{*}(n T) \rightarrow 0 \text {, weakly in } D(A) \text { as } n \rightarrow \infty \text {. }
$$

By (3.33), (3.35) and (3.37), it follows that,

$$
\left\{\begin{array}{l}
F^{*} \mathbf{q}_{n} \rightarrow F^{*} \mathbf{q}^{*}, \text { strongly in } L^{2}\left(\mathbb{R}^{+} ; U\right), \\
\mathbf{z}_{n} \rightarrow \mathbf{z}^{*} \text { strongly in } L^{2}\left(\mathbb{R}^{+} ; H\right)
\end{array}\right.
$$

Moreover, by (3.22) and (3.38) that

$$
\mathbf{z}_{n}(0) \rightarrow \mathbf{z}^{*}(0) \text { strongly in } V^{\prime} .
$$


Recall that $\{n\}$ is a subsequence of the initial sequence. Thus to conclude the claim, it suffices to show that the solution $\left(\mathbf{q}^{*}, \mathbf{z}^{*}\right)$ to Eq. (3.32) is unique. Let $\left(\overline{\mathbf{q}}^{*}, \mathbf{z}^{*}\right)$ be another solution to this system, we have

$$
\left\{\begin{array}{l}
\left(\mathbf{q}^{*}-\overline{\mathbf{q}^{*}}\right)^{\prime}(t)+\mathcal{A}^{*}\left(\mathbf{q}^{*}-\overline{\mathbf{q}^{*}}\right)=\mathbf{z}^{*}-\overline{\mathbf{z}^{*}} \\
\left(\mathbf{z}^{*}-\overline{\mathbf{z}^{*}}\right)^{\prime}(t)-\mathcal{A}\left(\mathbf{z}^{*}-\mathbf{z}^{*}\right)=F F^{*}\left(\mathbf{q}^{*}-\overline{\mathbf{q}^{*}}\right), \\
\left(\mathbf{q}^{*}-\overline{\mathbf{q}^{*}}\right)(0)=0,\left(\mathbf{z}^{*}-\overline{\mathbf{z}^{*}}\right)(\infty)=0
\end{array}\right.
$$

This yields that

$$
\int_{0}^{n T}\left(\left|\mathbf{z}^{*}-\overline{\mathbf{z}}^{*}\right|^{2}+\left|F^{*}\left(\mathbf{q}^{*}-\overline{\mathbf{q}}^{*}\right)\right|^{2}\right) d t=0 .
$$

Hence,

$$
\mathbf{z}^{*} \equiv \mathbf{z}^{*}, \mathbf{q}^{*} \equiv \mathbf{q}^{*} \text {. }
$$

We have therefore proven that $\left\{\nabla \varphi_{n}(\mathbf{x})\right\}$ is convergent in $V^{\prime}$.

Step 3: Convergence of $\left\{\varphi_{n}(\mathbf{x})-\varphi_{n}(0)\right\}$. We may infer as an intermediate step of the proof that

$$
\lim _{n \rightarrow \infty} \nabla \varphi_{n}(\mathbf{x})=\nu(\mathbf{x}) \in \partial \varphi^{0}(\mathbf{x}), \quad \forall \mathbf{x} \in V,
$$

where

$$
\varphi^{0}(\mathbf{x})=\lim _{n \rightarrow \infty}\left(\varphi_{n}(\mathbf{x})-\varphi_{n}(0)\right), \quad \forall \mathbf{x} \in V .
$$

We let $\varphi_{n}^{0}(\mathbf{x})=\varphi_{n}(\mathbf{x})-\varphi_{n}(0)$. We have

$$
\begin{aligned}
\varphi_{n}^{0}(\mathbf{x})= & \inf \left\{\int_{0}^{T}\left(\frac{1}{2}\left(\left|F^{*} \mathbf{q}(t)\right|^{2}+|\mathbf{z}(t)|^{2}\right)+\langle\mathbf{g}(t), \mathbf{q}(t)\rangle\right) d t+\varphi_{n-1}^{0}(\mathbf{q}(T))\right. \\
& \left.\mathbf{q}^{\prime}(t)+\mathcal{A}^{*} \mathbf{q}(t)=\mathbf{z}(t), \mathbf{q}(0)=\mathbf{x}, \mathbf{z} \in L^{2}(0, T ; H)\right\} \\
& +\varphi_{n-1}(0)-\varphi_{n}(0)
\end{aligned}
$$

This yields

$$
\begin{aligned}
\varphi_{n}^{0}(\mathbf{x}) \leq & \int_{0}^{T}\left(\frac{1}{2}\left(\left|F^{*} \mathbf{q}(t)\right|^{2}+|\mathbf{z}(t)|^{2}\right)+\langle\mathbf{g}(t), \mathbf{q}(t)\rangle\right) d t \\
& +\varphi_{n-1}^{0}(\mathbf{q}(T))+\varphi_{n-1}(0)-\varphi_{n}(0),
\end{aligned}
$$

for all admissible pair $(\mathbf{q}, \mathbf{z})$. We get therefore,

$$
\begin{aligned}
\varphi^{0}(\mathbf{x}) \leq & \inf \left\{\int_{0}^{T}\left(\frac{1}{2}\left(\left|F^{*} \mathbf{q}(t)\right|^{2}+|\mathbf{z}(t)|^{2}\right)+\langle\mathbf{g}(t), \mathbf{q}(t)\rangle\right) d t+\varphi^{0}(\mathbf{q}(T))\right. \\
& \left.\mathbf{q}^{\prime}(t)+\mathcal{A}^{*} \mathbf{q}(t)=\mathbf{z}(t), \mathbf{q}(0)=\mathbf{x}, \mathbf{z} \in L^{2}(0, T ; H)\right\} \\
& +\limsup _{n \rightarrow \infty}\left(\varphi_{n-1}(0)-\varphi_{n}(0)\right)
\end{aligned}
$$


On the other hand, there are $\left(\tilde{\mathbf{q}}_{n}, \tilde{\mathbf{z}}_{n}\right)$ such that

$$
\begin{aligned}
\varphi_{n}^{0}(\mathbf{x})= & \int_{0}^{T}\left(\frac{1}{2}\left(\left|F^{*} \tilde{\mathbf{q}}_{n}\right|^{2}+\left|\tilde{\mathbf{z}}_{n}\right|^{2}\right)+\left\langle\mathbf{g}(t), \tilde{\mathbf{q}}_{n}\right\rangle\right) d t \\
& +\varphi_{n-1}^{0}\left(\tilde{\mathbf{q}}_{n}(T)\right)+\varphi_{n-1}(0)-\varphi_{n}(0),
\end{aligned}
$$

and

$$
\tilde{\mathbf{q}}_{n}^{\prime}(t)+\mathcal{A}^{*} \tilde{\mathbf{q}}_{n}(t)=\tilde{\mathbf{z}}_{n}(t), \tilde{\mathbf{q}}_{n}(0)=\mathbf{x}
$$

Since

$$
\varphi_{n-1}^{0}\left(\tilde{\mathbf{q}}_{n}(T)\right) \geq \varphi_{n-1}^{0}(0)+\left\langle\partial \varphi_{n-1}^{0}(0), \tilde{\mathbf{q}}_{n}(T)\right\rangle .
$$

We see that $\left\{\tilde{\mathbf{z}}_{n}\right\}$ is bounded in $L^{2}(0, T ; H)$. Note also that by (3.14) we have

$$
\varphi_{n}(0) \geq \varphi_{n-1}(0)+\int_{(n-1) T}^{n T}\left(\frac{1}{2}\left(\left|F^{*} \mathbf{q}_{n}^{0}\right|^{2}+\left|\mathbf{z}_{n}^{0}\right|^{2}\right)+\left\langle\mathbf{g}(t), \mathbf{q}_{n}^{0}\right\rangle\right) d t,
$$

where $\left(\mathbf{q}_{n}^{0}, \mathbf{z}_{n}^{0}\right)$ is the optimal solution minimize the cost functional $\varphi_{n}(0)$. Then, by estimate (3.24), it follows that $\left\{\varphi_{n-1}(0)-\varphi_{n}(0)\right\}$ is bounded. Hence, on a subsequence, again denoted by $n$, we have,

$$
\left\{\begin{array}{l}
F^{*} \tilde{\mathbf{q}}_{n} \rightarrow F^{*} \tilde{\mathbf{q}}, \text { weakly in } L^{2}(0, T ; U), \\
\tilde{\mathbf{z}}_{n} \rightarrow \tilde{\mathbf{z}} \text { weakly in } L^{2}(0, T ; H), \\
\tilde{\mathbf{q}}_{n}(t) \rightarrow \tilde{\mathbf{q}}(t) \text { weakly in } V \text { for all } t \in[0, T] \\
\varphi_{n-1}(0)-\varphi_{n}(0) \rightarrow \mu .
\end{array}\right.
$$

We have also that

$$
\varphi_{n-1}^{0}(\tilde{\mathbf{q}}(T)) \leq \varphi_{n-1}^{0}\left(\tilde{\mathbf{q}}_{n}(T)\right)+\left\langle\partial \varphi_{n-1}^{0}(\tilde{\mathbf{q}}(T)), \tilde{\mathbf{q}}(T)-\tilde{\mathbf{q}}_{n}(T)\right\rangle .
$$

Since as seen earlier that $\left\{\partial \varphi_{n-1}^{0}(\tilde{\mathbf{q}}(T))\right\}$ is strongly convergent, we have

$$
\liminf _{n \rightarrow \infty} \varphi_{n-1}^{0}\left(\tilde{\mathbf{q}}_{n}(T)\right) \geq \varphi^{0}(\tilde{\mathbf{q}}(T)),
$$

and so by (3.48), (3.50) and (3.51) we see that

$$
\liminf _{n \rightarrow \infty} \varphi_{n}^{0}(\mathbf{x}) \geq \int_{0}^{T}\left(\frac{1}{2}\left(\left|F^{*} \tilde{\mathbf{q}}\right|^{2}+|\tilde{\mathbf{z}}|^{2}\right)+\langle\mathbf{g}(t), \tilde{\mathbf{q}}\rangle\right) d t+\varphi_{n}^{0}(\tilde{\mathbf{q}}(T))+\mu,
$$

Along with (3.47), the latter implies that

$$
\begin{aligned}
& \varphi^{0}(\mathbf{x}) \\
& =\lim _{n \rightarrow \infty} \varphi_{n}^{0}(\mathbf{x}) \\
& \quad=\inf \left\{\int_{0}^{T}\left(\frac{1}{2}\left(\left|F^{*} \mathbf{q}(t)\right|^{2}+|\mathbf{z}(t)|^{2}\right)+\langle\mathbf{g}(t), \mathbf{q}(t)\rangle\right) d t+\varphi^{0}(\mathbf{q}(T)) ;\right. \\
& \left.\quad \mathbf{q}^{\prime}(t)+\mathcal{A}^{*} \mathbf{q}(t)=\mathbf{z}(t), \mathbf{q}(0)=\mathbf{x}, \mathbf{z} \in L^{2}(0, T ; H)\right\}+\mu, \quad \forall \mathbf{x} \in V .
\end{aligned}
$$

Thus, the proof of Lemma 3.3 is complete. 
Continued: Define the function $\psi:[0, T] \times V \rightarrow \mathbb{R}$

$$
\begin{array}{r}
\psi(t, \mathbf{x})=\inf \left\{\int_{0}^{t}\left(\frac{1}{2}\left(\left|F^{*} \mathbf{q}(s)\right|^{2}+|\mathbf{z}(s)|^{2}\right)+\langle\mathbf{g}(s), \mathbf{q}(s)\rangle\right) d s+\varphi^{0}(\mathbf{q}(t))\right. \\
\left.\mathbf{q}^{\prime}(s)+\mathcal{A}^{*} \mathbf{q}(s)=\mathbf{z}(s), \mathbf{q}(0)=\mathbf{x}, \mathbf{z} \in L^{2}(0, T ; H)\right\},
\end{array}
$$

It is well known (see [10-12]) that $\psi$ is continuous, convex as function of $\mathbf{x}$ absolutely continuous in $t$ for each $\mathbf{x} \in D(A)$ and satisfies Eq. (3.3). By Lemma 3.3 we see also that $\psi(0, \mathbf{x})-\psi(T, \mathbf{x})=\mu$. Thus the proof of Theorem 3.1 is completed.

In the following, we give an application of the optimal synthesis results obtained above.

Consider now the stationary Navier-Stokes equation with homogeneous Dirichlet boundary condition

$$
\begin{cases}-\nu \triangle \mathbf{y}_{e}+\left(\mathbf{y}_{e} \cdot \nabla\right) \mathbf{y}_{e}+\nabla p=\mathbf{f}_{0} & \text { in } \Omega \\ \nabla \cdot \mathbf{y}_{e}=0 & \text { in } \Omega \\ \mathbf{y}_{e}=0 & \text { on } \partial \Omega .\end{cases}
$$

The existence, regularity and uniqueness of $\mathbf{y}_{e}$ was discussed in details in literature (see e.g. [3]). Everywhere in the following, we assume that the solution $\mathbf{y}_{e}$ is regular enough, for instance, $\mathbf{y}_{e} \in\left(W^{2, \infty}(\Omega)\right)^{2}$. This can be ensured with $\mathbf{f}_{0} \in\left(W^{1, \infty}(\Omega)\right)^{2}$. The solution to the following time-periodic equation

$$
\begin{cases}\mathbf{y}^{\prime}(t)-\nu \Delta \mathbf{y}+(\mathbf{y} \cdot \nabla) \mathbf{y}+\nabla p=\mathbf{f}_{0}+\mathbf{f}(t) & \text { in } \Omega, \\ \nabla \cdot \mathbf{y}= & \text { in } \Omega, \\ \mathbf{y}(0)=\mathbf{y}(T) & \text { in } \Omega, \\ \mathbf{y}=0 & \text { on } \partial \Omega\end{cases}
$$

may do not stay near the original solution no matter how small the perturbation $\mathbf{f}$ is. This means, the solution to the equation

$$
\begin{cases}\mathbf{y}^{\prime}(t)-\nu \triangle \mathbf{y}+\left(\mathbf{y}_{e} \cdot \nabla\right) \mathbf{y}+(\mathbf{y} \cdot \nabla) \mathbf{y}_{e}+(\mathbf{y} \cdot \nabla) \mathbf{y}+\nabla p=\mathbf{f}(t) & \text { in } \Omega \\ \nabla \cdot \mathbf{y}=0 & \text { in } \Omega, \\ \mathbf{y}(0)=\mathbf{y}(T) & \text { in } \Omega, \\ \mathbf{y}=0 & \text { on } \partial \Omega .\end{cases}
$$

is not continuously dependent on the outer force term. We shall prove below that we can put a boundary feedback control to overcome this defects for each perturbation $\mathbf{f}$ small enough. Still denote $\mathcal{A y}=A \mathbf{y}+P\left(\left(\mathbf{y}_{e} \cdot \nabla\right) \mathbf{y}+(\mathbf{y} \cdot \nabla) \mathbf{y}_{e}\right)$, and denote $B \mathbf{y}=P(\mathbf{y} \cdot \nabla) \mathbf{y}$. We have the following result.

Theorem 3.4. Assume that $\mathbf{y}_{e} \in\left(W^{2, \infty}(\Omega)\right)^{2}$. For all $0<\varepsilon<1 / 4$, There is a constant $\delta_{0}>0$ and a nondecreasing function $\eta$ from $\mathbb{R}^{+}$into itself, such that for each $\delta \in\left(0, \delta_{0}\right)$ and $\|f\|_{L^{2}\left(0, T ; V^{0}(\Omega)\right)} \leq \eta(\delta)$, there is a feedback boundary 
input $\mathbf{u}(t)=-F^{*} \psi_{\mathbf{y}}^{*}(t, \mathbf{y}(t))$ such that the periodic equation

$$
\left\{\begin{array}{l}
\frac{d \mathbf{y}}{d t}+\mathcal{A} \mathbf{y}+B \mathbf{y}=F \mathbf{u}+\mathbf{f} \\
\mathbf{y}(0)=\mathbf{y}(T)
\end{array}\right.
$$

admits a unique solution, in the set

$$
D_{\delta}=\left\{\mathbf{y} \in V^{3 / 2-\varepsilon, 3 / 4-\varepsilon / 2}\left(Q_{T}\right) ;\|\mathbf{y}\|_{V^{3 / 2-\varepsilon, 3 / 4-\varepsilon / 2}\left(Q_{T}\right)} \leq \delta, \mathbf{y}(0)=\mathbf{y}(T)\right\} .
$$

and the following estimate holds

$$
\|\mathbf{y}\|_{V^{3 / 2-\varepsilon, 3 / 4-\varepsilon / 2}\left(Q_{T}\right)} \leq C\|\mathbf{f}\|_{L^{2}\left(0, T ; V^{0}(\Omega)\right)} .
$$

Proof. For each $\mathbf{z} \in V^{3 / 2-\varepsilon, 3 / 4-\varepsilon / 2}\left(Q_{T}\right)$, we denote by $\mathbf{y}_{\mathbf{z}}$ the solution to the equation

$$
\frac{d \mathbf{y}}{d t}+\mathcal{A} \mathbf{y}+F F^{*} \psi_{\mathbf{y}}^{*}(t, \mathbf{y}(t))=-B \mathbf{z}+\mathbf{f}, \quad \mathbf{y}(0)=\mathbf{y}(T)
$$

We are going to prove that the mapping $\mathbf{M}: \mathbf{z} \mapsto \mathbf{y}_{\mathbf{z}}$ is a contraction in $D_{\delta}$.

(i) It follows from Theorems 2.2 and 3.2 that $\left(\mathbf{y}_{\mathbf{z}},-F^{*} \psi_{\mathbf{y}}^{*}\left(t, \mathbf{y}_{\mathbf{z}}(t)\right)\right)$ is optimal solution to OCP $(\mathrm{P})$, and we infer by Theorem 2.3 that

$$
\left\|\mathbf{y}_{\mathbf{z}}\right\|_{V^{3 / 2-\varepsilon, 3 / 4-\varepsilon / 2}\left(Q_{T}\right)} \leq\|-B \mathbf{z}+\mathbf{f}\|_{L^{2}\left(0, T ;\left(V^{2 \varepsilon}(\Omega)\right)^{\prime}\right)} .
$$

We can obtain that

$$
\begin{aligned}
& \left\|\mathbf{y}_{\mathbf{z}}\right\|_{V^{3 / 2-\varepsilon, 3 / 4-\varepsilon / 2}\left(Q_{T}\right)} \\
& \quad \leq C\left(\|\mathbf{f}\|_{L^{2}\left(0, T ;\left(V^{2 \varepsilon}(\Omega)\right)^{\prime}\right)}+\|-B \mathbf{z}\|_{L^{2}\left(0, T ;\left(V^{2 \varepsilon}(\Omega)\right)^{\prime}\right)}\right) \\
& \quad \leq C_{1}\left(\eta(\delta)+C_{2}\|\mathbf{z}\|_{V^{3 / 2-\varepsilon, 3 / 4-\varepsilon / 2}\left(Q_{T}\right)}^{2}\right) \leq \frac{3}{4} \delta+C_{1} C_{2} \delta^{2} \leq \delta
\end{aligned}
$$

for $\delta<\delta_{1}$, where $\delta_{1}$ sufficiently small. Thus, $\mathbf{M}$ is a mapping from $D_{\delta}$ into itself.

(ii)

$$
\begin{aligned}
\left\|\mathbf{y}_{\mathbf{z}_{\mathbf{1}}}-\mathbf{y}_{\mathbf{z}_{\mathbf{2}}}\right\|_{V^{3 / 2-\varepsilon, 3 / 4-\varepsilon / 2}\left(Q_{T}\right)} \leq C_{1}\left\|B \mathbf{z}_{\mathbf{1}}-B \mathbf{z}_{\mathbf{2}}\right\|_{L^{2}\left(0, T ;\left(V^{2 \varepsilon}(\Omega)\right)^{\prime}\right)} \\
\leq C_{1} C_{2}\left\|\mathbf{z}_{1}\right\|_{V^{3 / 2-\varepsilon, 3 / 4-\varepsilon / 2}\left(Q_{T}\right)}\left\|\mathbf{z}_{1}-\mathbf{z}_{\mathbf{2}}\right\|_{V^{3 / 2-\varepsilon, 3 / 4-\varepsilon / 2}\left(Q_{T}\right)} \\
\quad+C_{1} C_{2}\left\|\mathbf{z}_{2}\right\|_{V^{3 / 2-\varepsilon, 3 / 4-\varepsilon / 2}\left(Q_{T}\right)}\left\|\mathbf{z}_{1}-\mathbf{z}_{\mathbf{2}}\right\|_{V^{3 / 2-\varepsilon, 3 / 4-\varepsilon / 2}\left(Q_{T}\right)} \\
\leq 2 C_{1} C_{2}\left\|\mathbf{z}_{1}-\mathbf{z}_{\mathbf{2}}\right\|_{V^{3 / 2-\varepsilon, 3 / 4-\varepsilon / 2}\left(Q_{T}\right)} .
\end{aligned}
$$

Thus, we infer that

$$
\left\|\mathbf{y}_{\mathbf{z}_{1}}-\mathbf{y}_{\mathbf{z}_{\mathbf{2}}}\right\|_{V^{3 / 2-\varepsilon, 3 / 4-\varepsilon / 2}\left(Q_{T}\right)} \leq 2 C_{1} C_{2} \delta\left\|\mathbf{z}_{1}-\mathbf{z}_{\mathbf{2}}\right\|_{V^{3 / 2-\varepsilon, 3 / 4-\varepsilon / 2}\left(Q_{T}\right)} .
$$

Therefore, the mapping $M$ is a contraction in $D_{\delta}$ for $0<\delta<\delta_{0}$, where $\delta_{0}$ is a fixed sufficiently small positive number satisfying $\delta_{0}<\delta_{1}$. From the proof above, we can see that inequality (3.58) holds. 


\section{Appendix: Time-periodic Stokes equations}

Consider the Stokes equation

$$
\begin{cases}\frac{d \mathbf{y}}{d t}-\nu \triangle \mathbf{y}+\nabla p=0 & \text { in } \Omega \times(0, T), \\ \mathbf{y}(0)=\mathbf{y}(T) & \text { in } \Omega, \\ \nabla \cdot \mathbf{y}=0 & \text { in } \Omega \times(0, T), \\ \mathbf{y}=\mathbf{u} & \text { on } \partial \Omega \times(0, T) .\end{cases}
$$

The equation can be represented as the following abstract equation via Leray projection and Dirichlet map.

$$
\begin{aligned}
& P \mathbf{y}^{\prime}+\nu A P \mathbf{y}=F \mathbf{u}, \mathbf{y}(0)=\mathbf{y}(T) \\
& (I-P) \mathbf{y}=(I-P) D \gamma_{n} \mathbf{u} .
\end{aligned}
$$

Here $D$ is the operator defined in (1.2) with $k=0$.

Theorem 4.1. For each $\mathbf{u} \in L^{2}\left(0, T ; V^{0}(\Gamma)\right), \mathbf{u}(t, \mathbf{x})=\mathbf{u}(t+T, \mathbf{x})$, Eq. (4.1) admits a unique solution $\mathbf{y} \in L^{2}\left(0, T ; V^{1 / 2-\varepsilon}(\Omega)\right) \cap H^{1 / 4-\varepsilon}\left(0, T ; V^{0}(\Omega)\right)$.

Proof. It's known that Eq. (4.3) has a unique solution $\phi=(I-P) \mathbf{y}$ which is periodic and $\phi \in L^{2}\left(0, T ; V^{1 / 2-\varepsilon}\right) \cap H^{1 / 4-\varepsilon}\left(0, T ; V^{0}(\Gamma)\right)$. For the existence of solution of Eq. (4.2), we shall apply fixed point theorem for operator $\Upsilon$ : $P \mathbf{y}_{0} \mapsto P \mathbf{y}(T)$ on the subset of space $(D(A))^{\prime}$

$$
N_{r}=\left\{\mathbf{y}_{0} \in(D(A))^{\prime},\left\|\mathbf{y}_{0}\right\|_{(D(A))^{\prime}} \leq r\right\},
$$

where $\Upsilon\left(P \mathbf{y}_{0}\right)=P \mathbf{y}(T)$ is given by

$$
P \mathbf{y}(T)=e^{-\nu A T} P \mathbf{y}_{0}+\int_{0}^{T} e^{-\nu A(T-t)} F \mathbf{u}(t) d t
$$

(i) Consider the first part $e^{-A t} P \mathbf{y}_{0}$, it is the solution to the equation

$$
\mathbf{z}^{\prime}+\nu A \mathbf{z}=0, \mathbf{z}(0)=P \mathbf{y}_{0} .
$$

Multiplying Eq. (4.4) by $e^{\nu t} A^{-2} \mathbf{z}$, and integrating on $(0, T)$, we can get that

$$
\left\|e^{-\nu A T} P \mathbf{y}_{0}\right\|_{(D(A))^{\prime}}^{2} \leq e^{-\nu T}\left\|P \mathbf{y}_{0}\right\|_{(D(A))^{\prime}} .
$$

For the part $\int_{0}^{T} e^{-A(T-t)} F \mathbf{u}(t) d t$, we can get from the fact that $D P \mathbf{u} \in$ $L^{2}\left(0, T ; D\left(A^{1 / 4-\varepsilon}\right)\right), \forall \varepsilon \in(0,1 / 4)$, that

$$
\int_{0}^{t} e^{-\nu A(t-s)} F \mathbf{u}(s) d s \in L^{2}\left(0, T ; D\left(A^{1 / 4-\varepsilon}\right)\right) \cap H^{1}\left(0, T ; D\left(A^{3 / 4+\varepsilon}\right)^{\prime}\right) .
$$

It follows by interpolation inequality that

$$
\left\|\int_{0}^{T} e^{-\nu A(T-t)} F \mathbf{u}(t) d t\right\|_{D\left(A^{1 / 4+\varepsilon}\right)^{\prime}} \leq C\|\mathbf{u}\|_{L^{2}\left(0, T ; V^{0}(\Omega)\right)}
$$

Taking $r$ sufficient large, we can obtain that

$$
P \mathbf{y}(T)=\Upsilon\left(P \mathbf{y}_{0}\right) \in N_{r}
$$


(ii) Multiplying again Eq. (4.4) by $t A^{-1} \mathbf{z}$, and integrating on $(0, T)$, we can see that

$$
\left\|e^{-\nu A T} P \mathbf{y}_{0}\right\|_{\left(D\left(A^{1 / 2}\right)\right)^{\prime}}^{2} \leq e^{-\nu T}\left\|P \mathbf{y}_{0}\right\|_{(D(A))^{\prime}} .
$$

We deduce by (4.7) and (4.9) that

$$
\|P \mathbf{y}(T)\|_{\left(D\left(A^{1 / 2}\right)\right)^{\prime}}^{2} \leq C\left\|P \mathbf{y}_{0}\right\|_{(D(A))^{\prime}} .
$$

Since $\left(D\left(A^{1 / 2}\right)\right)^{\prime}$ is compact imbedded in $(D(A))^{\prime}$, it follows by Schaulder's fixed point theorem that equation (4.2) admits a solution. Moreover, we have by (4.7) that

$$
\begin{aligned}
\|P \mathbf{y}(0)\|_{\left(D\left(A^{1 / 4+\varepsilon}\right)\right)^{\prime}} & =\left\|\left(1-e^{-\nu A T}\right)^{-1} \int_{0}^{T} e^{-\nu A(T-t)} F \mathbf{u}(t) d t\right\|_{D\left(A^{1 / 4+\varepsilon}\right)^{\prime}} \\
& \leq C\|\mathbf{u}\|_{L^{2}\left(0, T ; V^{0}(\Omega)\right)}
\end{aligned}
$$

Hence

$$
\|P \mathbf{y}\|_{L^{2}\left(0, T ; D\left(A^{1 / 4-\varepsilon}\right)\right.} \leq C\|\mathbf{u}\|_{L^{2}\left(0, T ; V^{0}(\Omega)\right)} .
$$

It follows that $P \mathbf{y} \in H^{1}\left(0, T ; D\left(A^{3 / 4+\varepsilon}\right)^{\prime}\right)$, and we obtain by interpolation theory that

$$
\|P \mathbf{y}\|_{V^{1 / 2-\varepsilon, 1 / 4-\varepsilon / 2}\left(Q_{T}\right)} \leq C\|\mathbf{u}\|_{L^{2}\left(0, T ; V^{0}(\Omega)\right)} .
$$

This completes the proof of Theorem 4.1.

When the boundary input is more regular, we can also improve the regularity of the periodic solution to Eq. (4.2).

Theorem 4.2. When $u \in V^{s, s / 2}\left(\Sigma_{T}\right), 0 \leq s<1$, the solution to Eq. (4.2) satisfies the following estimate

$$
\|P \mathbf{y}\|_{V^{s+1 / 2-\varepsilon, s / 2+1 / 4-\varepsilon / 2}\left(Q_{T}\right)} \leq C\|\mathbf{u}\|_{V^{s, s / 2}\left(\Sigma_{T}\right)} .
$$

Proof. Taking first $\mathbf{u} \in V^{2,1}\left(\Sigma_{T}\right)$, Denote $\mathbf{w}=D \mathbf{u}$, then the function $\phi=$ $\mathbf{y}-\mathbf{w}$ satisfies the following equation

$$
\begin{cases}\frac{d \phi}{d t}-\nu \triangle \phi+\nabla p=\frac{d \mathbf{w}}{d t}-\nu \triangle \mathbf{w} & \text { in } \Omega \times(0, T), \\ \phi(0)=\phi(T) & \text { in } \Omega, \\ \nabla \cdot \phi=0 & \text { in } \Omega \times(0, T), \\ \phi=0 & \text { on } \partial \Omega \times(0, T) .\end{cases}
$$

Since $\mathbf{u} \in V^{2,1}\left(\Sigma_{T}\right)$, we know that $\mathbf{g}_{0}=\frac{d \mathbf{w}}{d t}-\nu \triangle \mathbf{w} \in L^{2}\left(0, T ; V^{1 / 2}(\Omega)\right)$, Eq. (4.15) can be written as

$$
\left\{\begin{array}{l}
\phi^{\prime}+\nu A \phi=\mathbf{g}, \\
\phi(0)=\phi(T),
\end{array}\right.
$$

where $\mathbf{g}=P \mathbf{g}_{0} \in L^{2}\left(0, T ; V_{n}^{1 / 2}(\Omega)\right)$. The energy estimate of Eq. (4.16) gives that

$$
\|\phi\|_{V^{2,1}\left(Q_{T}\right)} \leq C\|\mathbf{u}\|_{V^{2,1}\left(\Sigma_{T}\right)}
$$


Multiplying Eq. (4.16) by $t A^{3 / 2-\varepsilon} \phi$, and integrating on $(0, T)$, then we have that

$$
\left|A^{3 / 4-\varepsilon / 2} \phi(T)\right| \leq C\|\mathbf{u}\|_{V^{2,1}\left(\Sigma_{T}\right)}
$$

Thus $\phi(0)=\phi(T) \in D\left(A^{3 / 4-\varepsilon / 2}\right)$. On the other hand, $\|P D \mathbf{u}(0)\|_{V^{3 / 2}(\Omega)} \leq$ $C\|\mathbf{u}\|_{V^{2,1}\left(\Sigma_{T}\right)}$. Therefore,

$$
\operatorname{Py}(0)=P \mathbf{y}(T) \in V^{3 / 2-\varepsilon}(\Omega)
$$

Following the proof of Theorem 2.3 in Raymond [17], we can get that

$$
\|P \mathbf{y}\|_{V^{5 / 2-\varepsilon, 5 / 4-\varepsilon / 2}\left(Q_{T}\right)} \leq C\|\mathbf{u}\|_{V^{2,1}\left(\Sigma_{T}\right)} .
$$

Finally, we get with inequality (4.20), Theorem 4.1 and via interpolation theory that estimate (4.14) holds.

Remark 4.3. When we replace operator $\nu A$ by $\mathcal{A}_{k}$, we can follow the same arguments to get that Theorems 4.1 and 4.2 holds also in this case.

Now we are going to give some regularity results for periodic Stokes equations with homogeneous Dirichlet boundary condition but nonhomogeneous source term.

Theorem 4.4. If $\mathbf{f} \in L^{2}\left(0, T ;\left(V_{n}^{2 \varepsilon}(\Omega)\right)^{\prime}\right), 0<\varepsilon<1 / 4$, the solution $\mathbf{y}$ to the equation

$$
\mathbf{y}^{\prime}+\nu A \mathbf{y}=\mathbf{f}, \mathbf{y}(0)=\mathbf{y}(T)
$$

obeys

$$
\|\mathbf{y}\|_{V^{2-2 \varepsilon, 1-\varepsilon}\left(Q_{T}\right)} \leq C\|\mathbf{f}\|_{L^{2}\left(0, T ;\left(V_{n}^{2 \varepsilon}(\Omega)\right)^{\prime}\right)} .
$$

Proof. Taking first $\mathbf{f} \in L^{2}\left(0, T ; V_{n}^{0}(\Omega)\right)$, we know that

$$
\mathbf{y}(0)=\mathbf{y}(T)=\left(1-e^{-\nu A T}\right)^{-1} \int_{0}^{T} e^{-\nu A(T-t)} \mathbf{f}(t) d t \in V_{n}^{0}(\Omega) .
$$

Therefore, We can get easily by estimate of Eq. (4.21) that

$$
\|\mathbf{y}\|_{V^{2,1}\left(Q_{T}\right)} \leq C\|\mathbf{f}\|_{L^{2}\left(0, T ; V_{n}^{0}(\Omega)\right)} .
$$

Similarly, we can get that when $\mathbf{f} \in L^{2}\left(0, T ; V^{-1}(\Omega)\right)$,

$$
\|\mathbf{y}\|_{V^{1,1 / 2}\left(Q_{T}\right)} \leq C\|\mathbf{f}\|_{L^{2}\left(0, T ; V^{-1}(\Omega)\right)} .
$$

By interpolation, we can obtain estimate (4.22).

Theorem 4.5. If the function $\mathbf{y} \in V^{s, s / 2}\left(Q_{T}\right) \cap L^{2}\left(0, T ; V_{n}^{0}(\Omega)\right), 0 \leq s \leq 2$, then the solution $\Psi$ to the equation

$$
\Psi^{\prime}-\nu A \Psi=\mathbf{y}, \Psi(T)=\Psi(0)
$$

obeys

$$
\|\Psi\|_{V^{s+2, s / 2+1}\left(Q_{T}\right)} \leq C\|\mathbf{y}\|_{V^{s, s / 2}\left(Q_{T}\right)}
$$


Proof. We prove (4.24) first for $s=0$. Let $\mathbf{y} \in L^{2}\left(0, T ; V_{n}^{0}(\Omega)\right)$. The existence of periodic solution follows by the same arguments as in Theorem 4.1, and we can get

$$
\Psi(T)=\Psi(0)=\left(1-e^{-\nu A T}\right)^{-1} \int_{0}^{T} e^{-\nu A(T-t)} \mathbf{y}(t) d t \in V_{n}^{0}(\Omega) .
$$

We obtain therefore $\Psi \in L^{2}\left(0, T ; V_{0}^{1}(\Omega)\right) \cap W^{1,2}\left(0, T ; V^{-1}(\Omega)\right)$. Multiplying equation (4.23) by $(T-t) A \Psi$, and integrating on $(0, T)$, we obtain that $\Psi(T)=\Psi(0) \in V_{0}^{1}(\Omega)$, Hence $\Psi \in V^{2,1}\left(Q_{T}\right)$, and estimate (4.24) holds for $s=0$.

Now let us take $\mathbf{y} \in V^{2,1}\left(Q_{T}\right)$. We have that

$$
A \Psi(t)=e^{-\nu A(T-t)} A \Psi(T)+\int_{t}^{T} e^{-\nu A(s-t)} A \mathbf{y}(s) d s .
$$

Integrating on parts we obtain that

$$
A \Psi(t)=e^{-\nu A(T-t)} A \Psi(T)+\mathbf{y}(t)+\int_{t}^{T} e^{-\nu A(s-t)} \mathbf{y}^{\prime}(s) d s .
$$

Since $\mathbf{y} \in V^{2,1}\left(Q_{T}\right)$, we can get that

$$
A \Psi(T)=A \Psi(0)=\left(1-e^{-\nu A T}\right)^{-1}\left(\mathbf{y}(t)+\int_{0}^{T} e^{-\nu A t} \mathbf{y}^{\prime}(t) d s\right) \in V_{n}^{0}(\Omega)
$$

Moreover, $A \Psi(T)=A \Psi(0) \in V_{0}^{1}(\Omega)$, and

$$
\|A \Psi\|_{L^{2}\left(0, T ; V^{2}(\Omega)\right)} \leq\|y\|_{V^{2,1}\left(Q_{T}\right)},
$$

or

$$
\|\Psi\|_{L^{2}\left(0, T ; V^{4}(\Omega)\right)} \leq\|y\|_{V^{2,1}\left(Q_{T}\right)}
$$

Thus,

$$
\left\|\Psi^{\prime}\right\|_{L^{2}\left(0, T ; V^{2}(\Omega)\right)} \leq\|y\|_{V^{2,1}\left(Q_{T}\right)} .
$$

This shows that estimate (4.24) holds for $s=2$, and for $0<s<2$ it can be obtained by interpolation between the estimates obtained for $s=0$ and $s=2$.

Remark 4.6. When the operator $\nu A$ is replaced by $\mathcal{A}_{k}$ in Theorem 4.4 and replaced by $\mathcal{A}_{k}^{*}$ in Theorem 4.5 , by following the same arguments, we can prove that the above results also hold.

\section{Acknowledgments}

The author thanks the referees for their careful reading of this paper and valuable suggestions. 


\section{References}

[1] Barbu, V., Lasiecka, I., Triggiani, R.: Tangential boundary stabilization of Navier-Stokes equations. Mem. Am. Math. Soc. 181(852) (2006)

[2] Barbu, V.: Optimal control of Navier-Stokes equations with periodic inputs. Nonlinear Anal. Theory. Methods Appl. 31(1/2), 15-31 (1998)

[3] Temam, R.: Navier-Stokes Equations. North-Holland, Amsterdam (1984)

[4] Barbu, V.: Nonlinear Differential Equations of Monotone Type in Banach Spaces. Springer, Berlin (2010)

[5] Barbu, V.: Stabilization of Navier-Stokes Flows. Springer, New York (2011)

[6] Barbu, V., Lasiecka, I.: The unique continuation property of eigenfunctions to Stokes-Oseen operator is generic with respect to the coefficients. Nonlinear Anal. Theory. Methods Appl. 75(12), 4384-4397 (2012)

[7] Fabre, C., Lebeau, G: Prolongement unique des solutions de l'équation de Stokes. Commun. Partial Differ. Equ. 21, 573-596 (1996)

[8] Fabre, C., Lebeau, G.: Regularité et unicité pour le problème de Stokes. Commun. Partial Differ. Equ. 27, 437-475 (2002)

[9] Triggiani, R: Unique continuation from an arbitrary interior subdomain of the variable-coefficient Oseen equation. Nonlinear Anal. 71(10), 4967-4976 (2009)

[10] Barbu, V., Da Prato, G.: Hamilton-Jacobi equations in Hilbert spaces; variational and semigroup approach. Annali Mat. Pura Appl. CXLII, 303-349 (1985)

[11] Barbu, V., Da Prato, G.: Hamilton-Jacobi equations in Hilbert spaces, Pitman Research Notes in Mathematics, Boston (1983)

[12] Barbu, V., Precupanu, T.: Convexity and Optimization in Banach Spaces. D.Reidel, Dordrecht (1986)

[13] Barbu, V.: Optimal control of linear periodic resonant systems in Hilbert spaces. SIAM J. Control Optim. 35, 2137-2156 (1997)

[14] Barbu, V.: Optimal feedback controllers for periodic convex control problems. Nonlinear Differ. Equ. Appl. 3, 35-54 (1996)

[15] Barbu, V., Lasiecka, I., Triggiani, R.: Abstract settings for tangential boundary stabilization of Navier-Stokes equations by high- and low-gain feedback controllers. Nonlinear Anal. Theory Methods Appl. 64(12), 2704-2746 (2006)

[16] Bensoussan, A., Da Prato, G., Delfour, M., Mitter, S.K.: Representation and Control of Infinite Dimensional Control Systems. Birkhauser, Boston (1993)

[17] Raymond, J.P.: Stokes and Navier-Stokes equations with nonhomogeneous boundary conditions. Ann. Inst. H. Poincaré Anal. Non Linéaire 24, 921$951(2007)$ 
[18] Raymond, J.P.: Feedback boundary stabilization of the two-dimensional NavierStokes equations. SIAM J. Control Optim. 45, 790-828 (2006)

[19] Lions, J.L.: Non-homogeneous Boundary Value Problems and Applications, vol. 1. Springer, Berlin (1970)

[20] Raymond, J.P.: Feedback boundary stabilization of the three-dimensional incompressible Navier-Stokes equations. J. Math. Pures Appl. (9) 87(6), 627-669 (2007)

[21] Liu, H.: Boundary optimal control of time-periodic Stokes-Oseen flows. J. Optim. Theory Appl. 154(3), 1015-1035 (2012)

Hanbing Liu

School of Mathematics and Physics of China

University of Geoscience

Wuhan 430074

China

e-mail: hanbing272003@aliyun.com

Received: 6 April 2012.

Accepted: 25 December 2013. 